\title{
Equivariant formality of isotropy actions
}

\author{
Jeffrey D. Carlson* $\quad$ Chi-Kwong Fok ${ }^{\dagger}$
}

2nd March 2018

\begin{abstract}
We collect an assortment of results on equivariant formality of the isotropy action of a compact, connected Lie group $K$ on a compact homogeneous space $G / K$. If the isotropy action of $K$ on $G / K$ is equivariantly formal, then $G / K$ is formal in the sense of rational homotopy theory. This enables us to strengthen a theorem of Shiga-Takahashi to a characterization of equivariant formality in this case. Using a $\mathrm{K}$-theoretic analogue of equivariant formality introduced and shown by the second-named author to be equivalent to equivariant formality in the usual sense, we prove a representation-theoretic characterization for equivariant formality of the isotropy action and give a new, uniform proof of equivariant formality for some previously known classes of examples.
\end{abstract}

\section{Introduction}

Equivariant formality is an important property of certain topological group actions, first named by Goresky, Kottwitz, and MacPherson [GKM98, §1.2] but already identified as important as far back as the Borel Seminar on transformation groups $\left[\mathrm{BBF}^{+} 60, \mathrm{Ch}\right.$. XII], which allows, inter alia, the application of powerful integral localization formulas [BV82, AB84, JK95]. Broad classes of actions of especial interest are well known to be equivariantly formal, e.g., Hamiltonian torus actions on compact symplectic manifolds and linear algebraic torus actions on smooth complex projective varieties [GKM98, §1.2, Thm. 14.1]. It would be desirable to have more explicit characterizations of equivariant formality.

A natural place to start is with homogeneous spaces $G / K$, the orbits of Lie group actions. If $G$ is compact, the left translation action of $G$ on the right quotient $G / K$ is known to be equivariantly formal if and only if $K$ contains a maximal torus of $G$; in fact, for any subgroup $H$ of $G$ of rank higher than that of $K$, it is impossible that the restricted action $H$ on $G / K$ be equivariantly formal. The next natural task, then, and the subject of the present paper, is to characterize when the restricted isotropy action of $K$ on $G / K$ is equivariantly formal. We call a pair of compact, connected Lie groups $(G, K)$ an isotropy-formal pair when this occurs. Isotropy-formality has been studied by various authors, whose results we summarize here and in Section 2.1. All known

* J.D.C. was partially supported by a postdoctoral fellowship from the Instituto Nacional de Matemática Pura e Aplicada (IMPA) funded by the Coordenação de Aperfeiçoamento de Pessoal de Nível Superior (CAPES), and partially supported by the National Center for Theoretical Sciences (Taipei) during the conclusion of writing.

${ }^{\dagger}$ C.-K. F. was supported by a postdoctoral fellowship from the National Center for Theoretical Sciences.

2000 Mathematics Subject Classification: 55N25, 19L47, 57T15 (primary) 
results involve the notion of formality in the sense of rational homotopy theory. For instance, Shiga and Takahashi provided the following sufficient conditions for isotropy-formality.

Theorem 1.1 ([Shig6, Thm. A, Prop. 4.1][ST95, Thm. 2.2]). If $G / K$ is formal and $H^{*}(B G ; \mathbb{Q}) \longrightarrow$ $H^{*}(B G ; \mathbb{Q})^{N_{G}(K)}$ surjective, then $(G, K)$ is isotropy-formal. If $G / S$ is formal with $S$ a torus containing regular elements of $G$, then $(G, S)$ is isotropy-formal if and only if $H^{*}(B G ; \mathbb{Q}) \longrightarrow H^{*}(B S ; \mathbb{Q})^{N_{G}(S)}$ is surjective.

Recently, Goertsches and Noshari showed that an important class of formal homogeneous spaces is isotropy-formal.

Theorem 1.2 ([GN16][Goe12]). If $(G, K)$ is a (generalized) symmetric pair (see Example 2.3.3), then it is isotropy-formal.

The first-named author of the present paper also found the following characterization when $K \cong S^{1}$.

Theorem 1.3 ([Car14, Algorithm 1.4] and Example 2.3.2,4). Let G be a compact, connected Lie group and $S$ a circle subgroup. The pair $(G, S)$ is isotropy-formal if and only if

1. the restriction map $H^{1}(G ; \mathbb{Q}) \longrightarrow H^{1}(S ; \mathbb{Q})$ is surjective, or

2. the restriction map $H^{1}(G ; \mathbb{Q}) \longrightarrow H^{1}(S ; \mathbb{Q})$ is not surjective, and there exists $g \in G$ such that $g z g^{-1}=z^{-1}$ for all $z \in S$.

These cases are mutually exclusive.

We observe that in Theorem 1.I, formality is assumed, and the homogeneous spaces considered in Theorems 1.2 and 1.3 are formal. It turns out that this is no coincidence. We have the first main result of this paper as follows.

Theorem A. If a pair $(G, K)$ of compact, connected Lie groups is isotropy-formal, then $G / K$ is formal.

This enables us to obtain the following characterization of isotropy-formality, which is a strengthened version of Theorem 1.1.

Theorem 1.4. Let $(G, K)$ be a pair of compact, connected Lie groups and $S$ a maximal torus of $K$. Then $(G, K)$ is isotropy-formal if and only if it is formal and $H^{*}(B G ; \mathbb{Q}) \longrightarrow H^{*}(B S ; \mathbb{Q})^{N_{G}(S)}$ is surjective.

In the latter part of this paper we appeal to another tool, namely $K$-theory, to investigate isotropy-formality. Inspired by the notion of weak equivariant formality introduced by Harada and Landweber [HLo7], the second author of the present paper defined the related notion of rational K-theoretic equivariant formality (RKEF for short) [Fok17], which amounts to surjectivity of the forgetful map from equivariant $K$-theory with $\mathbb{Q}$ coefficients to ordinary $K$-theory. He also proved the following equivalence.

Theorem 1.5 (Fok [Fok17]). An action of a compact Lie group $G$ on a finite $C W$ complex $X$ is equivariantly formal if and only if it is rational K-theoretic equivariantly formal, if and only if it is $\mathbb{Q}$-weakly equivariantly formal in the sense of Definition 5.1. 
This equivalent formulation translates the problem of determining isotropy-formality to the context of $K$-theory. One advantage of this approach is that it becomes more straightforward to check if the forgetful map is surjective, since this amounts to determining if any vector bundle on a given homogeneous space can be equipped with an equivariant structure. Our second main result is a representation-theoretic characterization of isotropy-formality. Here, as usual, $R(\Gamma)$ is the complex representation ring of a group $\Gamma$, a ring $R$ is said to be regular at a prime ideal $I$ if the localization $R_{I}$ is a regular local ring, and covering homomorphism of topological groups is said to be central covering is its kernel lies in the center of its domain.

Theorem B. Let $(G, K)$ be a compact, connected pair, $\widetilde{G} \longrightarrow G$ any finite, central covering such that $\pi_{1} \tilde{G}$ is torsion-free, and $\widetilde{K}$ the identity component of the preimage of $K$ under the covering map. Then $(G, K)$ is isotropy-formal if and only if the image $R:=\widetilde{\imath}^{*} R(\widetilde{G}) \otimes \mathbb{Q}$ of the restriction map $\widetilde{i}^{*}: R(\widetilde{G}) \otimes \mathbb{Q} \longrightarrow$ $R(\widetilde{K}) \otimes \mathbb{Q}$ is regular at the restriction $I=\widetilde{\imath}^{*} I(\widetilde{G}) \otimes \mathbb{Q}$ of the augmentation ideal of $R(\widetilde{G}) \otimes \mathbb{Q}$.

Theorem B allows a new, uniform proof of isotropy-formality (and with Theorem A, of formality) for many known examples in the literature, including the generalized symmetric spaces of Theorem 1.2, for which the original proof by contrast requires a case-by-case analysis running through the classification theorem for such spaces.

There is a common philosophical thread underrunning these apparently disparate observations: isotropy-formality arises in situations where symmetry is maximized, as indicated by Theorem 1.2. In general, the map $H^{*}(B G ; \mathbb{Q}) \longrightarrow H^{*}(B S ; \mathbb{Q})^{N_{G}(S)}$ is not surjective, but if it is, morally speaking it is because the codomain $H(B S ; \mathbb{Q})^{N_{G}(S)}$ is minimized, meaning the size of the image of $N_{G}(S)$ in the automorphism group of $S$ is maximized. Similarly, the generators of the kernel of $i^{*}: R(G) \longrightarrow R(K)$ can be thought of as equations which cut out the subgroup $K$. The embedding of $K$ is symmetric if those equations are relatively simple, and regularity is the condition which describes this simplicity.

The organization of the paper is as follows. In Section 2 we provide relevant definitions and notation and review known examples and reduction results of isotropy-formality, then recall the algebraic definitions necessary to prove Theorem A. In Section 3 we proved Theorem A and some natural corollaries involving invariant theory. In Section 4 we illustrate by way of examples that none of the sufficient conditions for isotropy-formality discussed in previous sections implies the others and thus they are not extraneous. In Section 5, we recall the second-named author's definition of rational $\mathrm{K}$-theoretic equivariant formality [Fok17] and provide new proofs of Theorem 1.5 and some related results from the same paper. Section 6 concerns the rational K-theory ring structure of homogeneous spaces, which is parallel to the corresponding cohomological result given by Theorem 2.6. Finally in Section 7 we exploit rational $K$-theoretic equivariant formality to prove Theorem B and give an alternative, uniform proof of Theorem 1.2. Two computational examples are also given to demonstrate the utility of Theorem B.

Acknowledgments. J.D.C. gratefully acknowledges enlightening conversations with Omar Antolín Camarena, Matthias Franz, Oliver Goertsches, Steve Halperin, Friedrich Knop, Larry Smith, and Loring W. Tu. C.-K. F. would like to thank Loring W. Tu for bringing this problem to his attention, Reyer Sjamaar for his interest and Nan-Kuo Ho for her encouragement during the writing of this paper. 


\section{Definitions and background}

In this section we set up some notation and provide some background lemmata on equivariant cohomology, commutative graded algebras, as well as models for homogeneous spaces and homotopy biquotients.

\subsection{Equivariant formality and previous work}

In this subsection we summarize what is known about isotropy-formality, taking the opportunity to establish some notation and terminology along the way.

Definition 2.1. In all that follows, absent explicit indication to the contrary, cohomology of spaces is singular with rational coefficients. Given the continuous action of a group $G$ on a space $X$, we say the action is equivariantly formal when the fiber inclusion in the Borel fibration $X \rightarrow(X \times$ $E G) / G=: X_{G} \rightarrow B G$ induces a surjection $H_{G}^{*}(X) \longrightarrow H^{*} X$ from Borel equivariant cohomology to singular cohomology, or equivalently [Smi67, Prop. II.4.3, p. 89] if the projection makes $H_{G}^{*} X$ a free module over the coefficient ring $H_{G}^{*}:=H_{G}^{*}(\mathrm{pt})=H^{*}(B G)$.

When $G$ is a compact Lie group and $K$ a closed subgroup, we say for concision that $(G, K)$ is a compact pair. If in addition both groups are connected, we say $(G, K)$ is a compact, connected pair. The left action of $K$ on the right quotient $G / K$, given by $k \cdot g K=(k g) K$, is called the isotropy action. Later, we will have occation to use the observation $E K \times_{K} G / K$ is homotopy equivalent to the homotopy biquotient ${ }_{K} G_{K}$, the homotopy quotient of $G$ by the two-sided $K^{2}$-action given by $\left(k^{\prime}, k\right) \cdot g=k^{\prime} g k^{-1}$. If the isotropy action of $K$ on $G / K$ is equivariantly formal, we say the pair $(G, K)$ is isotropy-formal. We write $N=\pi_{0} N_{G}(K)=N_{G}(K) / Z_{G}(K)$ for the group of automorphisms of $K$ induced by conjugation, $W_{K}$ for the Weyl group of $K$, and $H_{K}^{N}:=\left(H_{K}^{*}\right)^{N_{G}(K)}$ for the invariant subring.

Existing work on isotropy-formality consists of an equivalent condition and several sufficient conditions. Theorem 1.1 is the most general of the sufficient conditions; we will improve this to an equivalence in Section 3. Other results have the flavor of reductions. For instance we can always replace $K$ by a torus and $G$ by the product of a simply-connected group and a torus.

Theorem 2.2 (Carlson [Car14, Thms. 1.1,2]). Let $(G, K)$ be a compact, connected pair, $S$ a maximal torus of $K$, and $\widetilde{S}$ the identity component of the preimage of $S$ under a finite covering $\widetilde{G}$ of $G$.

- The pair $(G, K)$ is isotropy-formal if and only if $(G, S)$ is.

- The pair $(G, S)$ is isotropy-formal if and only if $(\widetilde{G}, \widetilde{S})$ is.

The following are examples of isotropy-formal pairs from the literature.

Example 2.3. A compact pair $(G, K)$ is known to be isotropy-formal if any of the following conditions holds.

1. The ranks of $K$ and $G$ are equal (Goresky et al. [GKM98, Thm. 14.1(1)][Car15, Prop. 10.3.1]).

2. The restriction map $H^{*} G \longrightarrow H^{*} K$ is surjective (Shiga [Shig6, Cor. 4.2][Car15, Prop. 10.3.2]).

3. There is a Lie group automorphism $\sigma$ of $G$ such that $K=\left(G^{\sigma}\right)_{0}$ is the identity component of the fixed point subgroup (Goertsches-Noshari [Goe12, GN16]). In this case, we call $(G, K)$ a generalized symmetric pair. If $\sigma$ has finite order, $G / K$ is traditionally called a generalized symmetric space, and when $\sigma$ is an involution, a symmetric space. 
4. If the group $K \cong S^{1}$ is a circle and $H^{1} G \longrightarrow H^{1} S^{1}$ is not surjective (i.e., if Example 2.3.2 does not apply), then $\left(G, S^{1}\right)$ is isotropy-formal if and only if $|N|=2$, meaning conjugation by some $g \in G$ induces $z \mapsto z^{-1}$ on $S^{1}$ (Carlson [Car14, Thm. 7.2]).

\subsection{Algebraic notions}

To prove Theorem A, we will need to use an algebraic model of the map $G / K \longrightarrow{ }_{K} G / K$, so we briefly state what such models are and basic structural results we will call on.

Definition 2.4. We reserve the letters $P$ and $Q$ for positively-graded rational vector spaces concentrated respectively in odd and even degrees, writing $\wedge Q \otimes \wedge P$ for the free $\mathbb{Q}$-cGA on this space; $\wedge Q$ is a symmetric algebra and $\wedge P$ an exterior algebra. A pure Sullivan algebra is a $\mathbb{Q}$-CDGA of the form $(\wedge Q \otimes \wedge P, d)$ where $d$ is a derivation such that

$$
d P \leqslant \wedge Q \quad \text { and } \quad d(\wedge Q)=0 .^{1}
$$

A DGA $(A, d)$ is said to be formal if it can be joined by a zig-zag of DGA quasi-isomorphisms to its own cohomology $\left(H^{*}(A, d), 0\right)$, viewed as a DGA with differential zero. A $\mathbb{Q}$-CDGA $(\wedge V, d)$ is a model of a topological space $X$ if it can be joined by a zig-zag of DGA quasi-isomorphisms to the rational cochain algebra $C^{*}(X ; \mathbb{Q})$. A map $\left(\wedge V_{Y}, d\right) \longrightarrow\left(\wedge V_{X}, d\right)$ of models for $X$ and $Y$ is a model of $f: X \longrightarrow Y$ if the induced map in cohomology is $H^{*} f$. The space $X$ is said to be formal if $C^{*}(X ; \mathbb{Q})$ is formal. ${ }^{2}$

Pure Sullivan algebras have a structure theory which we will need. First note [Kos51, p. 76] [FHTo1, p. 435] the exterior degree on $\wedge P$ induces an exterior degree or lower grading $(\wedge Q \otimes \wedge P)_{p}:=$ $\wedge Q \otimes \wedge^{p} P$ which persists in cohomology. There are canonical CDGA maps

$$
(\wedge Q, 0) \stackrel{\chi}{\longrightarrow}(\wedge Q \otimes \wedge P, d) \stackrel{j}{\longrightarrow}(\wedge P, 0),
$$

respectively including $\wedge Q \otimes \mathbb{Q}$ in exterior degree zero and modding out $\left(\wedge^{\geqslant 1} Q\right)$. The image of $j^{*}$ is [Kos50, Thm. 13.2][And62, Thm. 10.4] the exterior algebra $\wedge \widehat{P}$ on the Samelson space $\widehat{P}:=P \cap \operatorname{im} j^{*}$. We write $\breve{P} \leqslant P$ for a graded linear complement to $\widehat{P}$.

Proposition 2.5 ([GHV76, Prop. II.4.IV, p. 71][Oni94, pp. 141, 210, 213]). The Samelson space $\widehat{P}$ is explicitly given as $\left\{z \in P: d z \in d P \cdot \wedge^{\geqslant 1} Q\right\}$, verbally, those generators $z$ such that $d z$ is redundant as a generator of $(d P)$. The complement $d \check{P}$ irredundantly generates the ideal $(d P)$.

The cochain maps $\chi$ and $j$ of (2.1) in fact determine a factorization of cochain complexes, yielding the main cohomological structure theorem.

Theorem 2.6 ([And62][GHV76, pp. 73, 83, 152][Oni94, p. 141, 211][Car15, Thm. 7.4.7,8]). Let $(\wedge Q \otimes \wedge P, d)$ be a pure Sullivan algebra. Then one has an algebra decomposition

$$
H^{*}(\wedge Q \otimes \wedge P) \cong H^{*}(\wedge Q \otimes \wedge \check{P}) \otimes \wedge \widehat{P} \cong(\wedge Q /(d P) \oplus \mathfrak{a}) \otimes \wedge \widehat{P},
$$

where $\mathfrak{a}=\bigoplus_{p \geqslant 1} H^{*}(\wedge Q \otimes \wedge \check{P})_{p}$ is the ideal of elements of positive exterior degree. The following are equivalent:

${ }^{1}$ The nilpotence condition [FHTo1, Def., p. 138] required of a Sullivan algebra follows automatically from the restrictions on the differential.

${ }^{2}$ The standard definition asks it be weakly equivalent through cDGAs to the minimal model of $C^{*}(X ; \mathbb{Q})$, but recent work of Saleh [Sali7] shows that for $\mathbb{F}$ of characteristic zero, these notions are equivalent. 
1. The ideal $\mathfrak{a}$ is 0 .

2. The algebra $(\wedge Q \otimes \wedge P, d)$ is formal.

3. The map $(\wedge Q \otimes \wedge P, d) \longrightarrow(\wedge Q /(d P) \otimes \wedge \widehat{P}, 0)$ is a quasi-isomorphism.

4. The ideal $(d P) \unlhd \wedge Q$ is generated by a regular sequence, or in other words $\wedge Q /(d P)$ is a complete intersection ring.

If $H^{*}(\wedge Q \otimes \wedge P)$ is finite-dimensional, one also has the equivalent condition

(v) $\operatorname{dim} P-\operatorname{dim} Q=\operatorname{dim} \widehat{P}$ (the inequality $\operatorname{dim} P-\operatorname{dim} Q \geqslant \operatorname{dim} \widehat{P}$ always holds).

A key lemma in the proof of Theorem A will involve identifying when the cohomology of such a model is free over a certain subring. For this we will need a bit more notation.

Definition 2.7. Given an augmented algebra $A \rightarrow k$ over a unital commutative ring $k$, we write $\tilde{A}$ for the augmentation ideal. When $A=\bigoplus_{n \geqslant 0} A_{n}$ is a commutative $\mathbb{N}$-graded algebra with $A_{0}=k$ (henceforth a connected $k$-CGA), we only ever consider that augmentation with kernel $\oplus_{n \geqslant 1} A_{n}$.

Given an $A$-module $M$, we define the module of indecomposables as the quotient

$$
Q_{A} M:=M / \tilde{A} \cdot M \cong \underset{A}{k \underset{A}{\otimes} M,}
$$

yielding a right exact functor from $A$-modules to $k$-modules which if $A$ is a connected CGA also reflects epimorphisms of nonnegatively graded $A$-modules [MM65, Prop. 3.8][NSo2, Cor. A.1.2]. In case $A \longrightarrow B$ is a map of augmented $k$-algebras, we also write $B / / A:=Q_{A} B=k \otimes_{A} B$. The quotient maps $q_{A, M}: M \longrightarrow Q_{A} M$ assemble into a natural transformation $q_{A}:$ id $\longrightarrow Q_{A}$. For $M=\widetilde{A}$, the indecomposables

$$
Q A:=\tilde{A} / \tilde{A} \cdot \tilde{A}
$$

yield a right exact functor from augmented $k$-algebras to $k$-modules [MM65, Prop. 3.11]. We write $q: A \rightarrow \widetilde{A} \rightarrow Q A$ as well for the projection to the indecomposables.

In case $A$ is a connected $k$-CGA and $M$ is $\mathbb{N}$-graded, liftings of indecomposables are generators in the sense that a $k$-linear section $\sigma$ of $q_{A, M}$ induces an $A$-module surjection

$$
\begin{aligned}
\psi(\sigma): A \underset{k}{\otimes} Q_{A} M & \longrightarrow M, \\
a \otimes \bar{m} & \longmapsto a \sigma(\bar{m}),
\end{aligned}
$$

since $Q_{A} \psi(\sigma)$ is an isomorphism; in words, im $\sigma$ is a $k$-module of $A$-module generators.

\section{Formality and reflections}

In this section we prove Theorem A and explore its consequences. 


\section{1. Construction of the model}

There are known models for $G / K$ and ${ }_{K} G / K$ which can be constructed using standard results about the rational homotopy theory of pullback fibrations [FHTo1, Props. 15.5,8]. The expected algebraic map between these two models the fiber inclusion $G / K \longleftrightarrow{ }_{K} G / K$.

Recall Hopf's theorem [Hopf41, Satz I, p. 23] that $H^{*} G$ is the exterior algebra on the subspace $P H^{*} G$ of primitive elements of the coproduct on $H^{*} G$ induced by the multiplication of $G$, and Borel's theorem [Bor53, Thm. 19.1] that the transgression $\tau: P H^{*} G \stackrel{\sim}{\longrightarrow} Q H_{G}^{*}$ in the Serre spectral sequence of the universal bundle is an isomorphism and $H_{G}^{*}$ can be seen as a polynomial algebra on a lift of $Q H_{G}^{*}$. The Cartan algebra [Car51, Thm. 5, p. 216][GHV76, Thm. 11.5.II, p. 462][Car15, Thm. 7.1.12] $]^{3}$ is the model of $G_{K}$ or equivalently ${ }^{4}$ of $G / K$ given by

$$
\left(H_{K}^{*} \otimes H^{*} G, d\right)
$$

for $d$ the unique derivation vanishing on $H_{K}^{*}$ and defined on $P H^{*} G$ as the composition

$$
d: P H^{*} G \stackrel{\tau}{\longrightarrow} Q H_{G}^{*} \stackrel{q^{\leftarrow}}{\longrightarrow} H_{G}^{*} \stackrel{\rho^{*}}{\longrightarrow} H_{K}^{*},
$$

where $q^{\leftarrow}: Q H_{G}^{*} \longmapsto H_{G}^{*}$ is some section of the projection $q$ to the indecomposables and $\rho=$ $B(K \hookrightarrow G)$.

Kapovitch [Kap, Prop. 1][FOTo8, \$3.4.2], building on work of Eschenburg [Esc92], discussed a pure Sullivan model for a biquotient, the orbit space of a free two-sided action on $G$ by a closed subgroup $U$ of $G^{2}$; our part is to observe this construction produces a model of the homotopy biquotient independent of freeness of the action. In the particular case $U=K^{2}$, the resulting model is

$$
\left(H_{K}^{*} \otimes H_{K}^{*} \otimes H^{*} G, \tilde{d}\right),
$$

where the derivation $\tilde{d}$ vanishes on $H_{K}^{*} \otimes H_{K}^{*}$ and sends a primitive $z \in P H^{*} G$ to

$$
\tilde{d} z:=1 \otimes d z-d z \otimes 1
$$

for $d$ the differential in the Cartan algebra just discussed.

By constructing a square of bundle maps connecting the pullback diagrams inducing these two models, one checks that $G_{K} \longrightarrow{ }_{K} G_{K}$ is modeled by reduction $\bmod H_{K}^{\geqslant 1} \otimes H_{K}^{*}$ :

$$
\begin{aligned}
\left(H_{K}^{*} \otimes H_{K}^{*} \otimes H^{*} G, \tilde{d}\right) & \longrightarrow\left(H_{K}^{*} \otimes H^{*} G, d\right), \\
H_{K}^{\geqslant 1} \otimes H_{K}^{*} \otimes H^{*} G & \longrightarrow 0, \\
1 \otimes x \otimes z & \longrightarrow x \otimes z .
\end{aligned}
$$

Notation 3.1. For brevity, we will write $H_{K}^{\otimes 2}:=H_{K}^{*} \otimes H_{K}^{*}$.

For future reference, note that the maps inducing the Cartan model fit into a fiber sequence

$$
K \longrightarrow G \longrightarrow G / K \longrightarrow B K \longrightarrow B G,
$$

thus yielding a cohomology sequence (cf. (2.I))

$$
H_{G}^{*} \stackrel{\rho^{*}}{\longrightarrow} H_{K}^{*} \stackrel{\chi^{*}}{\longrightarrow} H^{*}(G / K) \stackrel{j^{*}}{\longrightarrow} H^{*} G \stackrel{i^{*}}{\longrightarrow} H^{*} K .
$$

3 There is also a distinct differential form-based CDGA model of equivariant cohomology called the Cartan model. Cartan proved the result at hand using such a model, in the prototypical application of equivariant cohomology. Borel's proof in his thesis [Bor53, $\S \S 24-25]$ is a predecessor of the rational homotopy-theoretic argument.

4 It is important and not completely trivial that under this substitution the maps from $G$ and to $B K$ remain the expected ones up to homotopy [Car15, Sec. 7.1.1] but here we take this subtlety as dealt with. 


\subsection{The proof of Theorem A}

As isotropy-formality amounts to the request $H_{K}^{*} G_{K}$ be free over $H_{K}^{*}$, the proof is largely about characterizing free modules. First, note that not only do liftings of indecomposables characterize free modules, but any lifting will do.

Lemma 3.2. Let $k$ be a commutative ring, $A$ an augmented $k$-algebra, and $M$ an $A$-module. Then $M$ is a free $A$-module if and only if $Q_{A} M$ is a free $k$-module and for some section $\sigma$ of $q_{A, M}: M \longrightarrow Q_{A} M$, the map $\psi(\sigma)$ of (2.2) is an A-linear isomorphism. If $A$ is a connected $k$-CGA and $M$ is a finite free A-module, then $\psi(\sigma)$ is an isomorphism for any section $\sigma$.

Proof. If $Q_{A} M \cong k^{\oplus \lambda}$ for some cardinal $\lambda$ and there exists an $A$-module isomorphism $M \cong$ $A \otimes_{k} Q_{A} M$, then $M \cong A \otimes_{k} k^{\oplus \lambda} \cong A^{\oplus \lambda}$ is free over $A$. In the other direction, an $A$-module isomorphism $\phi: M \stackrel{\sim}{\longrightarrow} A^{\oplus \lambda}$ induces a $k$-module isomorphism $Q_{A} \phi: Q_{A} M \stackrel{\sim}{\longrightarrow} k^{\oplus \lambda}$, so $Q_{A} M$ is free over $k$ and we may identify $q_{A, M}: M \longrightarrow Q_{A} M$ with $q_{A, A^{\oplus \lambda}}: A^{\oplus \lambda} \longrightarrow k^{\oplus \lambda}$. Then the obvious section $\sigma: k^{\oplus \lambda} \longmapsto A^{\oplus \lambda}$ makes $\psi(\sigma)$ an $A$-linear isomorphism.

Supposing the additional hypotheses, for any section $\varsigma$ of $q_{A, A}$, the map $\psi(\varsigma)$ is a surjective $A$-module map from $A \otimes_{k} k^{\oplus \lambda} \cong A^{\oplus \lambda}$ to $A^{\oplus \lambda}$, and so is invertible [Vas69, Prop. 1.2][dJ' ${ }^{+}$, Lem. 10.15.4].

Second, base extensions along connected CGAs reflect freeness.

Lemma 3.3. Let $k$ a commutative ring, $A \leqslant B$ connected $k$-CGAs, and $M$ a finite $A$-module. If $B \underset{A}{\otimes} M$ is free over $B$, then $M$ is free over $A$.

Proof. Applying the right exact functor $-\otimes_{A} M$ to the exact sequence $0 \rightarrow \widetilde{B} \rightarrow B \rightarrow k \rightarrow 0$ of $A$ modules, one finds that $Q_{B}\left(B \otimes_{A} M\right) \cong k \otimes_{A} M$. From the assumption that $B \otimes_{A} M$ is free over $B$, it follows that $k \otimes_{A} M$ is free and finite over $k$, say on the basis $\left(1 \otimes x_{j}\right)$ for some elements $x_{j} \in M$, and since this basis is finite, it follows by Lemma 3.2 that extending the structure map $k \rightarrow B$ to a section $\sigma: k \otimes_{A} M \longmapsto B \otimes_{A} M$ of the projection $q_{B, B \otimes_{A} M}$ induces a $B$-module isomorphism ${ }^{5}$

$$
\begin{gathered}
B \underset{k}{\otimes}(k \underset{A}{\otimes} M) \stackrel{\psi}{\longrightarrow} B \underset{A}{\otimes} M, \\
\sum b_{j} \otimes\left(1 \otimes x_{j}\right) \\
\longmapsto \sum b_{j} \otimes x_{j} .
\end{gathered}
$$

The restriction of $\psi$ to $A \otimes_{k}\left(k \otimes_{A} M\right)$ factors through $A \otimes_{A} M$ as the surjective map in (2.2):

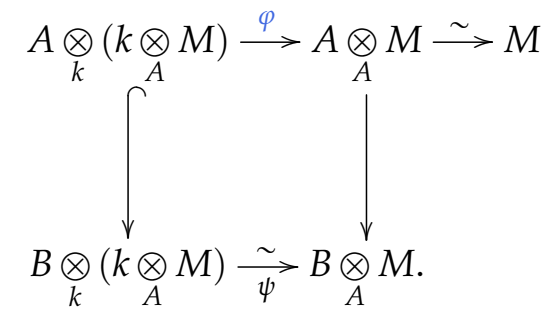

This map $\varphi$ must also be injective because the composition $A \otimes_{k} k \otimes_{A} M \longrightarrow B \otimes_{A} M$ along the lower-left is. The composition of $\varphi$ with the standard $A$-module isomorphism $A \otimes_{A} M \stackrel{\sim}{\longrightarrow} M$ presents $M$ as the free $A$-module on the basis $\left(x_{j}\right)$.

\footnotetext{
5 N.B. the dependence of this map on the arbitrary choice of basis; the "natural" guess taking $b \otimes(1 \otimes x) \mapsto b \otimes x$ for all $b$ and $x$ is ill-defined.
} 
We now apply these results to our model.

Proof of Theorem $A$. Recall that isotropy-formality is the two equivalent demands that the fiber inclusion in the Borel fibration $G_{K} \rightarrow{ }_{K} G_{K} \rightarrow B K$ induce a surjection $H_{K}^{*} G_{K} \longrightarrow H^{*}\left(G_{K}\right)$ in cohomology and the projection make $H_{K}^{*} G_{K}$ a free $H_{K}^{*}$-module. It follows from surjectivity that the images in $H^{*} G$ of the maps $H^{*}\left(H_{K}^{\otimes 2} \otimes H^{*} G\right) \rightarrow H^{*}\left(H_{K}^{*} \otimes H^{*} G\right) \rightarrow H^{*} G$ induced by the CDGA projections $H_{K}^{\otimes 2} \otimes H^{*} G \rightarrow H_{K}^{*} \otimes H^{*} G \rightarrow H^{*} G$ are equal, so the Samelson spaces of the two algebras agree. By Proposition 2.5, $d$ and $\tilde{d}$ respectively take a homogeneous basis of a linear complement $\check{P} \leqslant P=P H^{*} G$ to the Samelson space $\widehat{P}$ to irredundant sets of generators for $(d P)=(d \check{P}) \unlhd H_{K}^{*}$ and $(\tilde{d} P)=(\tilde{d} \check{P}) \unlhd H_{K}^{\otimes 2}$. Write $A \leqslant H_{K}^{*}$ for the subring generated by these chosen irredundant generators $d \check{P}$ of $(d P) \unlhd H_{K}^{*}$. The component of $H^{*}\left(H_{K}^{\otimes 2} \otimes H^{*} G\right)$ of exterior degree zero is

$$
H_{K}^{*} \otimes H_{K}^{*} /(\tilde{d} P)=H_{K}^{*} \otimes H_{K}^{*} /(\tilde{d} \check{P})=H_{K}^{*} \underset{A}{\otimes} H_{K}^{*}
$$

since $(\tilde{d} P)=(\tilde{d} \breve{P})$ and $\tilde{d} z=1 \otimes d z-d z \otimes 1$ for $z \in P$. Since the left $H_{K}^{*}$ factor of $H_{K}^{\otimes 2} \otimes H^{*} G$ lies in exterior grade zero, the left multiplication action of $H_{K}^{*}$ on $H^{*}\left(H_{K}^{\otimes 2} \otimes H^{*} G\right)$ preserves exterior degree, so as $H^{*}\left(H_{K}^{\otimes 2} \otimes H^{*} G\right)$ is a free $H_{K}^{*}$-module, its zero-graded component $H_{K}^{*} \otimes_{A} H_{K}^{*}$ is as well. Applying Lemma 3.3 in the case $k=\mathbb{Q}$ and $B=M=H_{K}^{*}$, we see the polynomial ring $H_{K}^{*}$ is free over $A$, and thus by a classical theorem of Macaulay [Smi95, Cor. 6.4.4], the $\operatorname{ring} A=\mathbb{Q}[d \check{P}]$ is itself polynomial and a basis of $d \check{P}$ forms a regular sequence in $H_{K}^{*}$. Thus $H_{K}^{*} / / H_{G}^{*}$ is a complete intersection ring, so $G / K$ is formal by Theorem 2.6 .

\subsection{Consequences of formality}

Isotropy-formality implies formality of the homotopy quotient as well.

Corollary 3.4. Let $(G, K)$ be an isotropy-formal compact, connected pair. Then ${ }_{K} G_{K}$ is itself formal. Particularly, there is an $\mathrm{H}_{\mathrm{K}}^{\otimes 2}$-algebra isomorphism

$$
H_{K}^{*}(G / K) \cong\left(H_{K}^{*} \underset{H_{G}^{*}}{\otimes} H_{K}^{*}\right) \otimes \wedge \widehat{P}
$$

where $\wedge \widehat{P}$ is the Samelson ring $\operatorname{im}\left(H^{*}(G / K) \longrightarrow H^{*} G\right)$.

Proof. The structure result was proven in the first-named author's thesis under an additional condition $G / K$ be formal [Car15, Thm. 11.1.1], but by Theorem A, this is already the case if $(G, K)$ is isotropy-formal. By Theorem 2.6, this implies ${ }_{K} G_{K}$ is formal.

Example 3.5. The converse to Corollary 3.4 does not hold. For example, consider the blockdiagonal inclusion of $\mathrm{SU}(3)^{2}$ in $\mathrm{SU}(6)$. In the Kapovitch model for $\mathrm{SU}_{(3)^{2}} \mathrm{SU}(6)_{\mathrm{SU}(3)^{2}}$, one can show the five differentials of a basis of $P H^{*} \mathrm{SU}(6)$ form a regular sequence in $H_{\mathrm{SU}(3)^{2}}^{\otimes 2}$, so by

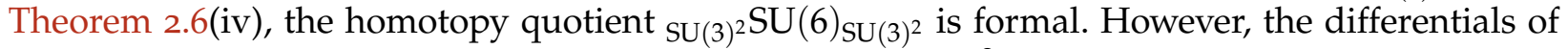
the same primitives in the Cartan algebra for $\mathrm{SU}(6) / \mathrm{SU}(3)^{2}$ are known not to form a regular sequence, so again by Theorem 2.6(iv), $\mathrm{SU}(6) / \mathrm{SU}(3)^{2}$ is not formal [Ama13, App. A] [GHV76, p. 486-488] and hence by Theorem A the pair $\left(\mathrm{SU}(6), \mathrm{SU}(3)^{2}\right)$ is not isotropy-formal.

Example 3.6. If both $G / K$ and ${ }_{K} G_{K}$ are formal, $(G, K)$ can still fail to be isotropy-formal. An example is given by $(G, K)=(\mathrm{SU}(7), \mathrm{SU}(3) \times \mathrm{SU}(4))$. The six differentials of a basis of $P H^{*} G$ in 
the algebra $\left(H_{K}^{\otimes 2} \otimes H^{*} G, \tilde{d}\right)$ can be shown to form a regular sequence in $H_{K}^{\otimes 2}$, so the Samelson subspace for ${ }_{K} G_{K}$ is zero-dimensional, but that for $G / K$ is 1 -dimensional, so $H_{K}^{*} G_{K} \rightarrow H^{*}(G / K)$ cannot be surjective.

Corollary 3.7. A compact, connected pair $(G, K)$ is isotropy-formal if and only if the image of $g: H_{K}^{*} G_{K} \rightarrow$ $H^{*}(G / K) \rightarrow H^{*} G$ meets $P H^{*} G$ in a space of dimension $\mathrm{rk} G-\mathrm{rk} K$.

Proof. If $(G, K)$ is isotropy-formal, then it is formal by Theorem $A$, and so by Theorem 2.6 we have $H^{*}(G / K) \cong\left(H_{K}^{*} / / H_{G}^{*}\right) \otimes \wedge \widehat{P}$ with $\operatorname{dim} \hat{P}=\operatorname{rk} G-\operatorname{rk} K$. Then since $H_{K}^{*} G_{K} \longrightarrow H^{*}(G / K)$ is surjective, its image particularly contains $\widehat{P}$, so im $g$ does as well. In the other direction, im $g$ lies in $\wedge \widehat{P}$, so if it meets $P H^{*} G$ in a space of dimension $\operatorname{rk} G-\operatorname{rk} K$, then $\operatorname{dim} \widehat{P}=\operatorname{rk} G-\operatorname{rk} K$, meaning $G / K$ is formal, and moreover, $\operatorname{im} g$ contains $\wedge \widehat{P}$. As $H_{K}^{*} \otimes_{H_{G}^{*}} H_{K}^{*} \longrightarrow H_{K}^{*} / / H_{G}^{*}$ as always surjective, the map $H_{K}^{*} G_{K} \longrightarrow H^{*}(G / K)$ is surjective as well and hence $(G, K)$ is isotropy-formal.

In particular, isotropy-formality of $(G, K)$ is equivalent to a statement about a certain privileged subspace of the Samelson space.

Definition 3.8. The transgression $\tau: P H^{*} G \stackrel{\sim}{\longrightarrow} Q H_{G}^{*}$ in the Serre spectral sequence of the universal bundle $E G \rightarrow B G$ induces a well-defined inverse function $\sigma: H_{G}^{*} \stackrel{q}{\rightarrow} Q H_{G}^{*} \stackrel{\sim}{\rightarrow} P H^{*} G \hookrightarrow H^{*} G$, the suspension. Here $q$ is as defined in Definition 2.7. We set $\widehat{P}_{0}:=\sigma \operatorname{ker}\left(\rho^{*}: H_{G}^{*} \rightarrow H_{K}^{*}\right) \leqslant P H^{*} G$.

It is clear from Proposition 2.5 that $\widehat{P}_{0}$ is contained in the Samelson space $\widehat{P}$ of the Cartan algebra, for since $q^{\leftarrow}$ is a section of $q$, for all $z \in \widehat{P}_{0}$ we have $d z=\rho^{*} q^{\leftarrow} \tau z \in \rho^{*}\left(\operatorname{ker} \rho^{*}+\operatorname{ker} q\right)=\rho^{*} H_{G}^{\geqslant 1}$. $\rho^{*} H_{G}^{\geqslant 1}$ Note that the Cartan algebra and Kapovitch model compute $H_{K}^{*}(G / K) \longrightarrow H^{*}(G / K)$ regardless of the choice of section $q^{\leftarrow}$ we employ in the definition of the differentials, so we are to choose this section at will.

Lemma 3.9. The space $\widehat{P}_{0}$ lies in the image of $g: H_{K}^{*} G_{K} \longrightarrow H^{*} G$.

Proof. Choose $q^{\leftarrow}$ such that $q^{\leftarrow} \tau \widehat{P}_{0}=q^{\leftarrow} q\left(\operatorname{ker} \rho^{*}\right)$ is contained in ker $\rho^{*}$ on the nose, rather than merely modulo the decomposables $H_{G}^{\geqslant 1} \cdot H_{G}^{\geqslant 1}$. Then, since the Cartan differential $d$ is $\rho^{*} q^{\leftarrow} \tau$ on $P H^{*} G$, we have $d \sigma \operatorname{ker} \rho^{*}=\rho^{*} q^{\leftarrow} q \operatorname{ker} \rho^{*} \leqslant \rho^{*}\left(\operatorname{ker} \rho^{*}\right)=0$, so that for $z \in \hat{P}_{0}$ we have $\tilde{d} z=$ $1 \otimes d z-d z \otimes 1=0$ in the Kapovitch model $\left(H_{K}^{\otimes 2} \otimes H^{*} G, \tilde{d}\right)$.

Proposition 3.10. A compact, connected pair $(G, K)$ is isotropy-formal if and only if $\operatorname{dim} q\left(\operatorname{ker} \rho^{*}\right)=$ $\operatorname{dim} \widehat{P}_{0}=\operatorname{rk} G-\operatorname{rk} K$.

Proof. By Lemma 3.9, if $\operatorname{dim} \widehat{P}_{0}=\mathrm{rk} G-\mathrm{rk} K$, then $(G, K)$ is isotropy-formal by Corollary 3.7. On the other hand if $(G, K)$ is isotropy-formal, then by the enhanced Shiga-Takahashi theorem 1.4 and Proposition 3.12 to follow, $\varkappa: H_{G}^{*} \longrightarrow H_{S}^{N}$ is surjective and $H_{S}^{N}$ is a polynomial ring on the same number of indeterminates as generate $H_{S}^{*}$ and $H_{K^{\prime}}^{*}$ the vector space $H_{K}^{*} / / H_{S}^{N}$ being finite-dimensional. It follows

$$
\operatorname{dim} \operatorname{ker} Q \varkappa=\operatorname{dim} Q H_{G}^{*}-\operatorname{dim} Q H_{S}^{N}=\operatorname{rk} G-\operatorname{rk} K .
$$

But $\operatorname{ker} Q \varkappa=q(\operatorname{ker} \varkappa)$ : an element of the former is $q(x)$ for some $x \in H_{G}^{*}$ such that $\varkappa(x) \in$ $\left(H_{S}^{\geqslant 1}\right)^{N} \cdot\left(H_{S}^{\geqslant 1}\right)^{N}=\varkappa\left(H_{G}^{\geqslant 1} \cdot H_{G}^{\geqslant 1}\right)$, meaning there is $y \in H_{G}^{\geqslant 1} \cdot H_{G}^{\geqslant 1}$ such that $x-y \in$ ker $\varkappa$ and hence $q(x)=q(x-y) \in q(\operatorname{ker} \varkappa)$. Finally, $\rho^{*}$ factors as $H_{G}^{*} \varkappa_{\varkappa} H_{S}^{N} \nrightarrow H_{K}^{*}$ since $W_{K} \leqslant N$, so ker $\varkappa=\operatorname{ker} \rho^{*}$. 
Later, in Section 7, we will find a K-theoretic analogue of Proposition 3.10, namely Proposition 7.15, naturally constructing a class of vector bundles which admit equivariant lifts. As formality is one of the conditions of the Shiga-Takahashi theorem 1.1, our Theorem A allows one to substantially strengthen it.

Theorem 1.4. Let $(G, K)$ be a pair of compact, connected Lie groups and $S$ a maximal torus of $K$. Then $(G, K)$ is isotropy-formal if and only if it is formal and $H^{*}(B G ; \mathbb{Q}) \longrightarrow H^{*}(B S ; \mathbb{Q})^{N_{G}(S)}$ is surjective.

Proof. The "if" direction is Shiga's original result. The other direction follows from the ShigaTakahashi theorem and Theorem A. The regular element hypothesis ${ }^{6}$ turns out not to play an essential role in the proof of Theorem I.I and can be omitted.

Remark 3.11. Theorem 1.4 can actually be strengthened to replace surjectivity of $H_{G}^{*} \longrightarrow H_{S}^{N_{G}(S)}$ with that of $H_{G}^{*} \longrightarrow H_{K}^{N_{G}(K)}$, but doing so would take us far afield, as it seems to require an alternate proof of the original Shiga-Takahashi theorem.

We also have an invariant-theoretic formulation. Recall that we write $N=\pi_{0} N_{G}(S)$.

Proposition 3.12. Suppose a compact, connected pair $(G, K)$ is such that $H_{G}^{*} \longrightarrow H_{S}^{N}$ is surjective. Then $(G, K)$ is isotropy-formal if and only if it is formal and $\pi_{0} N_{G}(S)$ acts on the Lie algebra 5 of $S$ as a reflection group.

Proof. Note that $H_{S}^{*} / / H_{G}^{*}=H_{S}^{*} / / H_{S}^{N}$ by the assumption $H_{G}^{*} \longrightarrow H_{S}^{N}$. By Theorem 1.4, $(G, K)$ is isotropy-formal if and only if $G / K$ is formal, which by Theorem 2.6 occurs if and only if $H_{S}^{*} / / H_{G}^{*}=H_{S}^{*} / / H_{S}^{N}$ is a complete intersection ring, which by the Chevalley-Shepherd-Todd theorem [Kaneo1, p. 192] occurs if and only if $N$ is a reflection group.

\section{Examples and counterexamples}

This section is devoted to showing the irredundancy of the three conditions for isotropy-formality discussed in the previous subsection. These non-formal examples are mostly to be found in a section in Greub et al. [GHV76, Ch. XI, §5].7 Example 4.4 for $n=5$ is due to Borel and according to Paul Baum has been circulating since at least the 1960s.

Example 4.1. That $N$ be a reflection group does not ensure $G / K$ be formal nor $H_{G}^{*} \longrightarrow H_{S}^{N}$ surjective.

The pair $(\mathrm{U}(5), S)$, where $S$ is the four-dimensional subtorus $\left\{\operatorname{diag}\left(z^{4}, w^{3}, \zeta^{2}, z w \zeta, \vartheta\right)\right\}$ of the diagonal torus, has $N=1$ a reflection group (generated by zero reflections). A computation with the computer algebra system Macaulay2 shows the regular sequence condition of Theorem 2.6(iv) is violated, so $\mathrm{U}(5) / S$ is not formal. One also incidentally sees that $\operatorname{dim}_{\mathbb{Q}} H_{S}^{*} / / H_{G}^{*}=22$, meaning particularly that the map $H_{G}^{*} \longrightarrow H_{S}^{N}=H_{S}^{*}$ is not surjective. The relevant code is in an auxiliary file hosted on the first-named author's website [Cari6].

Example 4.2. That $(G, K)$ be formal and $N$ a reflection group (even the Weyl group of $K$ ) does not ensure $H_{G}^{*} \longrightarrow H_{S}^{N}$ be surjective.

\footnotetext{
${ }^{6}$ A regular element of a Lie group $G$ is one lying in a unique maximal torus.

7 See the paper of Amann [Ama13] for many more, and Onishchik [Onig4, §13.4] for another family. Note these examples are all of deficiency $\operatorname{dim} P-\operatorname{dim} Q-\operatorname{dim} \widehat{P}=1$.
} 
The pair $(\mathrm{SU}(7), \mathrm{SU}(3) \times \mathrm{SU}(4))$ from Example 3.6 is formal but not isotropy-formal, and $N=S_{3} \times S_{4}=W_{K}$ is a reflection group. As another example, consider a compact, connected pair $(G, S)$, where $S \cong S^{1}$ is a circle not reflected by the larger group, such as $\left\{\operatorname{diag}\left(z, z, z^{-2}\right)\right\}$ or $\left\{\operatorname{diag}\left(z, z^{2}, z^{-3}\right)\right\}$ in $\mathrm{SU}(3)$. Then $N=1$ and $(G, S)$ is formal [Car14, App. A], but by Example 2.3 .4 , the pair $(G, S)$ is not isotropy-formal.

Example 4.3. That $H_{G}^{*} \longrightarrow H_{K}^{N}$ be surjective does not ensure $N$ be a reflection group or $(G, K)$ formal.

Consider again the block-diagonal inclusion of $K=\mathrm{SU}(3)^{2}$ in $G=\mathrm{SU}(6)$ from Example 3.5 . We already saw $G / K$ is not formal. The Weyl group $W_{G}=S_{6}$ permutes the six coordinates of the diagonal maximal torus $T$ and the stabilizer $N$ of $S=T \cap K$ in $W_{G}$ is generated by $W_{K}=S_{3} \times S_{3}$ and $\varepsilon=(14)(25)(36)$, which is not a product of reflections of $\mathfrak{s}$. But $H_{G}^{*} \longrightarrow H_{S}^{N}=H_{K}^{\langle\varepsilon\rangle}$ is indeed surjective; explicitly, it is

$$
\mathbb{Q}\left[c_{2}, c_{3}, c_{4}, c_{5}, c_{6}\right] \longrightarrow\left(\mathbb{Q}\left[c_{2}, c_{3}\right] \otimes \mathbb{Q}\left[c_{2}^{\prime}, c_{3}^{\prime}\right]\right)^{\langle\varepsilon\rangle}=\mathbb{Q}\left[c_{2}+c_{2}^{\prime}, c_{3}+c_{3}^{\prime}, c_{2} c_{2}^{\prime}, c_{2} c_{3}^{\prime}+c_{2}^{\prime} c_{3}, c_{3} c_{3}^{\prime}\right]
$$

each displayed generator on the left being sent to the corresponding generator on the right.

Example 4.4. None of the conditions need hold at all.

Consider the natural embedding of $K=\operatorname{SU}(n)$ in $G=\operatorname{Sp}(n)$. The Weyl group $W_{G}=\{ \pm 1\}^{n} \rtimes$ $S_{n}$ acts on the diagonal maximal torus $T$ of $G$ by permutating and inverting coordinates, and the condition det $=1$ on $S=T \cap K$ yields $N=S_{n} \times\langle\varepsilon\rangle$, where $\varepsilon\left(t_{1}, \ldots, t_{n}\right)=\left(t_{1}^{-1}, \ldots, t_{n}^{-1}\right)$. It can be shown $\mathrm{Sp}(n) / \mathrm{SU}(n)$ is formal [GHV76, §11.15, pp. 488-90] (in fact, isotropy-formal) if and only if $n \leqslant 4$ and that $N$ is a reflection group in exactly those cases. The map $H_{\mathrm{Sp}(n)}^{*} \longrightarrow H_{\mathrm{SU}(n)}^{\langle\varepsilon\rangle}$ on the other hand is surjective if and only if $n \leqslant 5$. Thus $(\operatorname{Sp}(5), \operatorname{SU}(5))$ shows, like Example 4.3 , that $H_{G}^{*} \longrightarrow H_{S}^{N}$ can be surjective without $N$ being a reflection group or $G / S$ formal. The larger pairs $(\mathrm{Sp}(n), \mathrm{SU}(n))$ provide examples in which none of the three conditions figuring in Proposition 3.12 hold.

In summary,

- None of the three conditions need hold.

- None of the three conditions alone implies isotropy-formality.

- That $N$ be a reflection group implies neither of the other conditions.

- That $H_{G}^{*} \longrightarrow H_{S}^{N}$ be surjective implies neither of the other conditions.

- That $(G, K)$ be formal does not imply that $H_{G}^{*} \longrightarrow H_{S}^{N}$ be surjective.

\section{Comparing equivariant $K$-theory and cohomology}

In this section, we switch to another tool, namely (equivariant) $K$-theory, and develop $K$-theoretic results necessary to study the equivariant formality of homogeneous spaces later on.

Definition 5.1 ([HLo7, Def. 4.1]). Let $k$ be a torsion-free ring. Write $K^{*}(-; k)$ and $K_{G}^{*}(-; k)$ for the unique additive ( $G$-equivariant) cohomology theories extending to all $(G-) C W$ complexes the functors defined by $X \longmapsto K^{*} X \otimes k$ and $(G \frown X) \longmapsto K_{G}^{*}(X) \otimes k$ on finite complexes. We also write 
$R(G ; k)=R(G) \otimes k$ and $I(G ; k)=I(G) \otimes k$ for the extended representation ring and augmentation ideal. The forgetful map $K_{G}^{*}(X) \longrightarrow K^{*}(X)$ factors through the homomorphism

$$
k \underset{R(G ; k)}{\otimes} K_{G}^{*}(X ; k) \longrightarrow K^{*}(X ; k) .
$$

We say the action is k-weakly equivariantly formal if the latter map is an isomorphism. Following Harada-Landweber, we simply say the action is weakly equivariantly formal in the case $k=\mathbb{Z}$.

As we defined equivariant formality as the surjectivity of $H_{G}^{*} X \longrightarrow H^{*} X$, for us the more natural analogue in $K$-theory is the following definition due to the second-named author.

Definition 5.2 (Fok [Foki7]). A G-action on a space $X$ is said to be rational K-theoretic equivariantly formal (or just $R K E F$ ) if the forgetful map

$$
f: K_{G}^{*}(X ; \mathbb{Q}) \longrightarrow K^{*}(X ; \mathbb{Q})
$$

is surjective.

This condition admits a natural interpretation in terms of vector bundles: for every vector bundle $V$ over $X$ or its suspension $\Sigma X$, there are natural numbers $m, n$ such that $V^{\oplus m} \oplus \underline{\mathbb{C}}^{n}$ admits an equivariant $G$-structure.

\section{1. The equivariant Chern character}

Recall [AH61, §2.4] that the Chern character induces a natural $\mathbb{Z} / 2$-graded ring homomorphism ch: $K^{*}(X) \longrightarrow H^{* *} X:=\prod_{n} H^{n} X$ which becomes an isomorphism $K^{*}(X ; \mathbb{Q}) \stackrel{\sim}{\longrightarrow} H^{*} X$ on finite $\mathrm{CW}$ complexes $X$. Analogously, a $G$-equivariant vector bundle $V \rightarrow X$ induces a vector bundle $V_{G} \rightarrow X_{G}$ of homotopy quotients and a class $\operatorname{ch}_{G}(V):=\operatorname{ch}\left(V_{G}\right) \in H^{* *} X_{G}=: H_{G}^{* *} X$, the equivariant Chern character of $V$. As homotopy quotients respect the semiring operations, these classes collate into a natural $\mathbb{Z} / 2$-graded ring homomorphism

$$
\mathrm{ch}_{G}: K_{G}^{*}(X) \longrightarrow H_{G}^{* *} X
$$

which, though typically far from surjective, nevertheless prescribes $H_{G}^{* *} X$.

Theorem 5.3. Let $X$ be a compact $G$-space such that $K_{G}^{*} X$ is a finite $R(G)$-module. The equivariant Chern character induces $\mathbb{Z} / 2$-graded ring isomorphisms

$$
K_{G}^{*}(X ; \mathbb{Q}) \stackrel{\sim}{\longrightarrow} H_{G}^{* *} X \stackrel{\sim}{\longleftarrow} H_{G}^{* *} \underset{R(G)}{\otimes} K_{G}^{*} X
$$

natural in such $X$, where $K_{G}^{*}(X ; \mathbb{Q})$ is the completion of $K_{G}^{*}(X ; \mathbb{Q})$ with respect to the augmentation ideal $I(G ; \mathbb{Q}) \triangleleft R(G ; \mathbb{Q})$. This isomorphism preserves the augmentation to $\mathbb{Q}$.

Proof. Let $E_{n} G \rightarrow B_{n} G$ be the restrictions of $E G \rightarrow B G$ over $C W n$-skeleta of $B G$ and $X_{n, G}$ the compact spaces $E_{n} G \times_{G} X$. The equivariant Chern character $K_{G}^{*}(X) \rightarrow K^{*}\left(X_{G}\right) \rightarrow H^{* *} X_{G}$ induces pro-ring maps $\left(K_{G}^{*}(X) / I(G)^{n} \cdot K_{G}^{*}(X)\right) \rightarrow\left(K^{*}\left(X_{n, G}\right)\right) \stackrel{\text { ch }}{\rightarrow}\left(H^{*} X_{n, G}\right)$. By the Atiyah-Segal completion theorem [AS69, Cor. 2.1], the second map is a pro-ring isomorphism, and since all objects 
are finite $R(G)$-modules and the $X_{n, G}$ are homotopy equivalent to finite CW complexes, tensoring with $\mathbb{Q}$ yields pro-ring isomorphisms

$$
\left(K_{G}^{*}(X ; \mathbb{Q}) / I(G ; \mathbb{Q})^{n} \cdot K_{G}^{*}(X ; \mathbb{Q})\right) \stackrel{\sim}{\rightarrow}\left(K^{*}\left(X_{n, G} ; \mathbb{Q}\right)\right) \stackrel{\sim}{\rightarrow}\left(H^{*} X_{n, G}\right) .
$$

Since the first inverse system clearly satisfies the Mittag-Leffler condition and thus has trivial $\lim ^{1}$, so do the other two, and hence [Mil62, Lem. 2] taking limits yields isomorphisms $K_{G}^{*}(X ; \mathbb{Q}) \wedge \stackrel{\sim}{\rightarrow} K^{*}\left(X_{G} ; \mathbb{Q}\right) \stackrel{\sim}{\rightarrow} H^{* *} X_{G}$. Particularly, $R(G ; \mathbb{Q})^{\wedge} \cong H_{G}^{* *}$, and since $R(G)$ is Noetherian, by the finiteness assumption it follows [AM69, Prop. 10.13]

$$
H_{G}^{* *} X \cong H_{G}^{* *} \underset{R(G ; \mathbb{Q})}{\otimes} K_{G}^{*}(X ; \mathbb{Q}) \cong H_{G}^{* *} \underset{R(G)}{\otimes} K_{G}^{*} X
$$

Naturality follows from cellular approximation and naturality of the Chern character.

Remark 5.4. The first isomorphism in Theorem 5.3 has been stated in the mathematical physics literature and cited as "a completion theorem of Atiyah and Segal" [Valo8, Thm. 6.7], though technically it is a distinct corollary requiring some argument like the above.

We need one more commutative algebra lemma before the main result of this section.

Lemma 5.5. Let $A$ be a Noetherian ring, $M$ a finite $A$-module, $\widehat{A}$ and $\widehat{M}$ the respective completions of $A$ and $M$ with respect to an ideal $\mathfrak{a}$ of $A$, and $N$ an $\widehat{A}$-module. Then the induced A-module structure on $N$ is such that $N \underset{A}{\otimes} M \cong N \underset{\widehat{A}}{\otimes} \widehat{M}$.

Proof. The finiteness assumptions make the natural map $\widehat{A} \otimes_{A} M \longrightarrow \widehat{M}$ a $\widehat{A}$-module isomorphism [AM69, Prop. 10.13]. Thence $\underset{\widehat{A}}{\otimes} \widehat{M} \cong \underset{\widehat{A}}{\otimes} \underset{A}{\widehat{A}} \underset{A}{\otimes} M \cong \underset{A}{\otimes} M$.

The main result of this section is the following result of the second-named author, published with a different proof in earlier work.

Theorem 1.5 (Fok [Fok17]). An action of a compact Lie group $G$ on a finite $C W$ complex $X$ is equivariantly formal if and only if it is rational K-theoretic equivariantly formal, if and only if it is $\mathbb{Q}$-weakly equivariantly formal in the sense of Definition 5.1.

Proof. Consider the commutative diagram

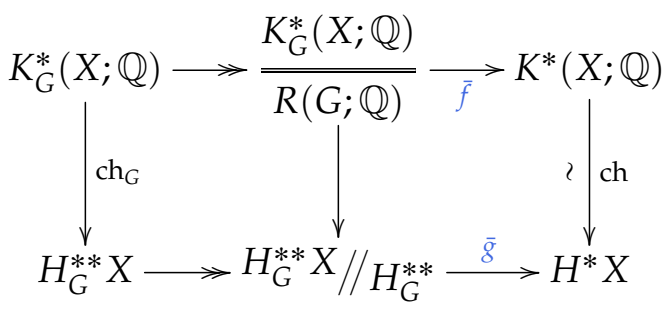

factoring the forgetful map $f: K_{G}^{*}(X ; \mathbb{Q}) \longrightarrow K^{*}(X ; \mathbb{Q})$ and restriction $g: H_{G}^{* *} X \longrightarrow H^{*} X$. By Lemma 5.5 , applied to the case where $A=R(G ; \mathbb{Q}), M=K_{G}^{*}(X ; \mathbb{Q})$, and $N=\mathbb{Q}$, and the isomorphism $R(G ; \mathbb{Q})^{\wedge} \cong H_{G}^{* *} X$ from Theorem $5 \cdot 3$, the middle vertical map is an isomorphism. Thus we can identify $\bar{f}$ with $\bar{g}$. But the Serre spectral sequence of $X \rightarrow X_{G} \rightarrow B G$ collapses at $E_{2}$ if and only if $g$ or equivalently $\bar{g}$ is surjective, implying the kernel of $g$ is the ideal generated by $H_{G}^{\geqslant 1}$, so that $\bar{g}$ is an isomorphism. Thus

$\bar{f}$ is surjective $\Longleftrightarrow \bar{g}$ is surjective $\Longleftrightarrow \bar{g}$ is an isomorphism $\Longleftrightarrow \bar{f}$ is an isomorphism. 
By definition, the action is cohomologically equivariantly formal if and only if $g$ or equivalently $\bar{g}$ is surjective, rationally K-theoretically equivariantly formal if and only if $f$ or equivalently $\bar{f}$ is surjective, and $\mathbb{Q}$-weakly equivariantly formal if and only if $\bar{f}$ is an isomorphism.

\section{The K-theory of compact homogeneous spaces}

In this section, we assume that $G$ and $K$ are compact, connected Lie groups unless otherwise specified. The structure theorem 2.6, as applied to the Cartan algebra $\left(H_{K}^{*} \otimes H^{*} G, d\right)$ computing $H^{*}(G / K)$, carries over to the context of $K$-theory by means of the Chern character.

For the right action of $K$ on $G$, the structure map $\alpha: R(K) \rightarrow K_{K}^{0} G \stackrel{\sim}{\rightarrow} K^{0}(G / K)$ making $K_{K}^{*}(G)$ an $R(K)$-algebra sends a $K$-representation $\rho$ to the class of the associated vector bundle $G \times{ }_{K} V_{\rho}$. Recall that $K^{*}(G ; \mathbb{Q})$ is a Hopf algebra with comultiplication induced by the group multiplication, so it makes sense to speak of its space of primitives and we can define a $K$-theoretic Samelson space in analogy with the definition in Section 2.2.

Definition 6.1. The K-theoretic Samelson space of $G / K$, denoted by $\hat{P}$, is the space $P K^{*}(G ; \mathbb{Q}) \cap$ im $j^{*}$ of primitive elements of $K^{*}(G ; \mathbb{Q})$ lying in the image of the pullback $j^{*}: K^{*}(G / K ; \mathbb{Q}) \longrightarrow$ $K^{*}(G ; \mathbb{Q})$. We also use $\widehat{P}$ to denote a preimage in $K^{*}(G / K ; \mathbb{Q})$ under the map $j^{*}$ if there is no danger of confusion, given that $j^{*}$ maps isomorphically from this preimage onto $\hat{P}$. We call $\wedge \widehat{P} \leqslant K^{*}(G ; \mathbb{Q})\left(\right.$ or $\left.\wedge \widehat{P} \leqslant K^{*}(G / K ; \mathbb{Q})\right)$ the $K$-theoretic Samelson ring.

Theorem 6.2. Let $(G, K)$ be a compact, connected pair. The maps of (6.1) below induce a ring isomorphism

$$
K^{*}(G / K ; \mathbb{Q}) \cong(R(K ; \mathbb{Q}) / / R(G ; \mathbb{Q}) \oplus \mathfrak{a}) \otimes \wedge \widehat{P} .
$$

Here $R(K) / / R(G)$ is the image of $\alpha: R(K) \longrightarrow K^{*}(G / K)$, the subalgebra $\wedge \widehat{P}$ is taken isomorphically onto the image of $j^{*}: K^{*}(G / K ; \mathbb{Q}) \longrightarrow K^{*}(G ; \mathbb{Q})$, and the summand $\mathfrak{a}$ is an ideal of the other tensor factor. We always have $\operatorname{dim} \hat{P} \leqslant \operatorname{rk} G-\operatorname{rk} K$. The space $G / K$ is formal if and only if $\operatorname{dim} \hat{P}=\operatorname{rk} G-\operatorname{rk} K$, if and only if $\mathfrak{a}=0$, if and only if $R(K ; \mathbb{Q}) / / R(G ; \mathbb{Q})$ is a complete intersection ring.

The Chern character isomorphism as applied to Theorem 2.6 in the special case of the Cartan algebra $\left(H_{K}^{*} \otimes H^{*} G, d\right)$ already yields a decomposition of the isomorphism type indicated, so the new content is only that the three factors can be identified as claimed. The proof uses a commutative diagram

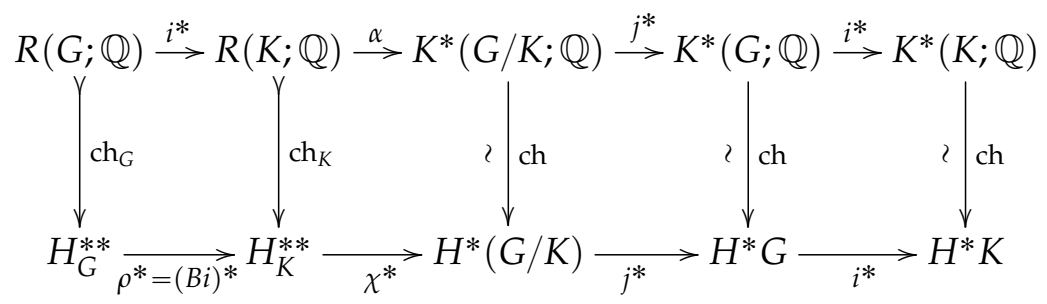

induced by (3.1) and expanding (3.2).

Proof. To see that the image of $\alpha$ is $R(K ; \mathbb{Q}) / / R(G ; \mathbb{Q})$, we show that ch $\circ \alpha$ induces an isomorphism $\mathbb{Q} \otimes_{R(G ; \mathbb{Q})} R(K ; \mathbb{Q}) \stackrel{\sim}{\longrightarrow} \mathbb{Q} \otimes_{H_{C}^{* *}} H_{K}^{* *}$. By Theorem $5 \cdot 3, H_{K}^{* *}$ is the completion of $R(K ; \mathbb{Q})$ with respect to $I(K ; \mathbb{Q})$, and since the $I(K)$-adic and $I(G)$-adic topologies on $R(K)$ agree [Seg68, Cor. 3.9], $H_{K}^{* *}$ 
is also the completion with respect to $i^{*} I(G ; \mathbb{Q})$. Thus Lemma 5.5 applied in the case $A=R(G ; \mathbb{Q})$ and $M=R(K ; \mathbb{Q})$ and $N=\mathbb{Q}$ yields the isomorphism.

The identification of the exterior factor follows from the third square in (6.1). That im $j^{*}$ is also generated by primitives of $K(G ; \mathbb{Q})$ follows from the fact that $K^{*}(G ; \mathbb{Q}) \stackrel{\sim}{\longrightarrow} H^{*} G$ is an isomorphism of Hopf algebras [Hod67, pf., Cor. II.2.3].

Remark 6.3. Much of Theorem 6.2 was already known; Snaith mentioned in passing in 1971 that the Künneth spectral sequence beginning at $E_{2}=\operatorname{Tor}_{R(G)}(\mathbb{Z}, R(K))$, which Hodgkin had already shown to converge to $K^{*}(G / K)$, collapses at $E_{2}$ modulo torsion [Hod75, Thm. 8.1(ii)][Sna71, p. 562]. This implies our theorem on the level of $R(G ; \mathbb{Q})$-modules. In fact, the sequence collapses even with torsion if $\pi_{1} G$ is torsion-free [Min75, Thm. 2.1]. With the additional assumption $\pi_{1} G$ is torsion-free, this yields explicit expressions with $\mathbb{Z}$ coefficients for the nice cases enumerated in Example 2.3: Minami proved the expected result in the case $R(G) \longrightarrow R(K)$ is surjective [Min75, Prop. 4.1] and also, building on work of Harris, in the case $G / K$ is a symmetric space [Har68, Min75], and of Pittie in the case where the ranks of $G$ and $K$ are equal [Pit72].

\section{Isotropy formality through the lens of $K$-theory}

This section is devoted to studying isotropy-formality of homogeneous spaces by means of $K$ theory. Using the equivalence of cohomological equivariant formality and $K$-theoretic equivariant formality asserted by Theorem 1.5 and the description of the $K$-theory of homogeneous spaces given by Theorem 6.2, we will give alternative proofs of the isotropy-formality of some previously known examples. Furthermore, by unraveling Theorem 1.5 in the context of homogeneous spaces, we give another characterization of isotropy-formality in terms of representation theory. Before doing so, we give a K-theoretic version of the Shiga-Takahashi conditions for isotropy-formality analogous to Theorem 1.4. One might be tempted to simply replace the condition that $\rho^{*}: H_{G}^{*} \longrightarrow$ $H_{S}^{N}$ be surjective by the condition that the restriction map $i^{*}: R(G ; \mathbb{Q}) \rightarrow R(S ; \mathbb{Q})^{N}$ be surjective, but it turns out that this naive translation is incorrect (see Example 7.18). Nevertheless, a slight modification admits a $\mathrm{K}$-theoretic analogue.

Proposition 7.1. Let $(G, K)$ be a compact, connected pair and $N=\pi_{0} N_{G}(K)$.

1. The restriction map $\rho^{*}: H_{G}^{*} \longrightarrow H_{K}^{N}$ is surjective if and only if $\left(H_{K}^{*} / / H_{G}^{*}\right)^{N} \cong \mathbb{Q}$.

2. $(G, K)$ is isotropy-formal if and only if it is formal and $(R(K ; \mathbb{Q}) / / R(G ; \mathbb{Q}))^{N} \cong \mathbb{Q}$.

Proof. 1. By the graded Nakayama lemma [NSo2, Prop. A.1.1], $\rho^{*}$ is surjective if and only if $H_{K}^{N} / / H_{G}^{*} \cong \mathbb{Q}$. But $H_{K}^{N} / / H_{G}^{*}=\left(H_{K}^{*} / / H_{G}^{*}\right)^{N}$, for if $a \in H_{K}^{*}$ represents an $N$-invariant element of $H_{K}^{*} / / H_{G^{\prime}}^{*}$, then so also does the $N$-average $\frac{1}{|N|} \sum_{w \in N} w^{*} a$.

2. This follows from Theorem 1.4 and the isomorphism $H_{K}^{*} / / H_{G}^{*} \cong R(K ; \mathbb{Q}) / / R(G ; \mathbb{Q})$ in the proof of Theorem 6.2.

\subsection{Isotropy-formality of pairs arising from Lie group automorphisms}

Throughout this section, we assume that both $G$ and $K$ are compact, connected Lie groups. We want to find a $K$-theoretic necessary condition for isotropy-formality of a pair $(G, K)$. By 
Theorem 1.5 , this is equivalent to the forgetful map $K_{K}^{*}(G / K ; \mathbb{Q}) \longrightarrow K^{*}(G / K ; \mathbb{Q})$ being surjective. We know from Theorem A that if $(G, K)$ is to be isotropy-formal, $G / K$ must be formal, and hence by Theorem 6.2 that its rational $K$-theory is the tensor product of a complete intersection $\operatorname{ring} R(K ; \mathbb{Q}) / / R(G ; \mathbb{Q})$ and an exterior factor $\wedge \widehat{P}$. The factor $R(K ; \mathbb{Q}) / / R(G ; \mathbb{Q})$ is in the image of $\alpha: R(K ; \mathbb{Q}) \rightarrow K^{*}(G / K ; \mathbb{Q})$, and so in the $\mathbb{Q}$-linear span of the classes represented by associated bundles $G \times{ }_{K} V_{\rho} \rightarrow G / K$ of $K$-representations $\rho$. These bundles are equivariant with respect to the action induced by the left multiplication of $K$ on $G$, so all elements of the factor $R(K ; \mathbb{Q}) / / R(G ; \mathbb{Q})$ admit equivariant lifts in $K_{K}^{*}(G / K ; \mathbb{Q})$. To determine if the forgetful map is surjective, then, it remains only to see if elements of the Samelson space $\widehat{P}$ generating the other tensor factor admit equivariant lifts as well.

We digress for a while to state foundational results on the (equivariant) K-theory of $G$.

Definition 7.2. 1. ([Har13, p. 172][BZoo, §2.3]) Let $A$ be a ring and $B$ an $A$-algebra. The module of Kähler differentials of $B$ over $A$ is the quotient $\Omega_{B / A}^{1}$ of the free $B$-module on the symbols $\{d b: b \in B\}$ by the $B$-submodule generated by the sets $\{d a: a \in A\}$, $\left\{d\left(b+b^{\prime}\right)-d b-d b^{\prime}: b, b^{\prime} \in B\right\}$, and $\left\{d\left(b b^{\prime}\right)-b d b^{\prime}-b^{\prime} d b: b, b^{\prime} \in B\right\}$. The commutative graded algebra of Grothendieck differentials $\Omega_{B / A}^{*}$ is defined to be the exterior $B$-algebra $\wedge_{B} \Omega_{B / A}^{1}=\oplus_{p=0}^{\infty} \bigwedge_{B}^{p} \Omega_{B / A}^{1}$.

2. ([Hod67, §I.4][BZoo, §3][FokI4, Defs. 2.2,5]) Let $G$ be a compact Lie group. Then $\delta: R(G) \longrightarrow$ $K^{-1}(G)$ is the map which sends a complex G-representation $\rho$ with underlying vector space $V$ to the class represented by the complex of vector bundles

$$
\begin{aligned}
0 \rightarrow G \times \mathbb{R} \times V \longrightarrow G \times \mathbb{R} \times V \rightarrow 0, & \\
(g, t, v) & \longmapsto \begin{cases}(g, t,-t \rho(g) v) & \text { if } t \geqslant 0, \\
(g, t, t v) & \text { if } t \leqslant 0 .\end{cases}
\end{aligned}
$$

Let $G$ act on itself by conjugation and $K_{G^{\text {Ad }}}^{*}(G)$ denote the equivariant $K$-theory of $G$ with respect to this action. ${ }^{8}$ For any subgroup $K$ of $G$ we may define a map $\delta_{K}: R(G) \longrightarrow K_{K^{\text {Ad }}}^{*}(G)$ similarly: $\delta_{K}(\rho)$ is the above complex of vector bundles equipped with the $K$-action given by $k \cdot(g, t, v)=\left(k g k^{-1}, t, \rho(k) v\right)$.

Theorem 7.3 (Hodgkin [Hod67, p. 8 ff.]). Let G be a compact, connected Lie group. The map $\delta: R(G) \longrightarrow$ $K^{-1}(G)$ is a $\mathbb{Z}$-linear derivation, or in other words satisfies

$$
\delta\left(\rho_{1} \otimes \rho_{2}\right)=\operatorname{dim}\left(\rho_{1}\right) \delta\left(\rho_{2}\right)+\operatorname{dim}\left(\rho_{2}\right) \delta\left(\rho_{1}\right) .
$$

Consequently $\delta(\mathbb{Z})=\delta\left(I(G)^{2}\right)=0$ and so $\delta$ factors through a group homomorphism $Q R(G) \longrightarrow K^{-1}(G)$. We have im $\delta \otimes \mathbb{Q}=P K^{*}(G ; \mathbb{Q})$, inducing an isomorphism of Hopf algebras

$$
\bar{\varphi}: \bigwedge_{\mathbb{Q}}[\operatorname{im} \delta \otimes \mathbb{Q}] \stackrel{\sim}{\longrightarrow} K^{*}(G ; \mathbb{Q}) .
$$

Theorem 7.4 ([BZoo, §3]). 1. The map $\delta_{G}$ in Definition 7.2.2 is a derivation of $R(G)$ taking values in the $R(G)$-module $K^{-1}\left(G^{\text {Ad }}\right)$; i.e., $\delta_{G}$ satisfies

$$
\delta_{G}\left(\rho_{1} \otimes \rho_{2}\right)=\rho_{1} \delta_{G}\left(\rho_{2}\right)+\rho_{2} \delta_{G}\left(\rho_{1}\right) .
$$

\footnotetext{
${ }^{8}$ The superscript "Ad" emphasizes the action is by conjugation.
} 
2. Let $G$ be a compact, connected Lie group with torsion-free fundamental group and $\Omega_{R(G) / \mathbb{Z}}^{*}$ the ring of Grothendieck differentials of $R(G)$ over $\mathbb{Z}$. There is an $R(G)$-algebra isomorphism

$$
\varphi: \Omega_{R(G) / \mathbb{Z}}^{*} \longrightarrow K_{G^{A d}}^{*}(G)
$$

defined by $\varphi\left(\rho_{V}\right):=[G \times V] \in K_{G}^{0}(G)$ and $\varphi\left(d \rho_{V}\right):=\delta_{G}\left(\rho_{V}\right)$, where $G$ acts on $G \times V$ by $g_{0} \cdot\left(g_{1}, v\right)=\left(g_{0} g_{1} g_{0}^{-1}, \rho_{V}\left(g_{0}\right) v\right)$.

Remark 7.5. We will use later the observation that Hodgkin's isomorphism $\bar{\varphi}$ in Theorem $7 \cdot 3$ is precisely the reduction mod $I(G)$ of the Brylinski-Zhang isomorphism $\varphi$ in Theorem 7.4. Note also that for each closed subgroup $K$ of $G$, the map $\delta_{K}: R(G) \longrightarrow K_{K^{\text {Ad }}}^{-1}(G)$ also satisfies $\delta_{K}\left(\rho_{1} \otimes \rho_{2}\right)=i^{*}\left(\rho_{1}\right) \delta_{K}\left(\rho_{2}\right)+i^{*}\left(\rho_{2}\right) \delta_{K}\left(\rho_{1}\right)$ and reduction $\bmod I(K)$ sends the image of $\delta_{K}$ to that of $\delta$.

Definition 7.6. An element $\rho \in \operatorname{ker}\left(i^{*}: R(G) \rightarrow R(K)\right)$ may be written as the formal difference $\rho_{1}-\rho_{2} \in R(G)$ of complex $G$-representations whose restrictions to $K$ agree. If $V$ is the vector space underlying the restricted representation, then we define maps $\delta^{G / K}: \operatorname{ker} i^{*} \rightarrow K^{-1}(G / K)$ and $\delta_{K}^{G / K}: \operatorname{ker} i^{*} \rightarrow K_{K}^{-1}(G / K)$ sending $\rho$ to the class represented by the complex of vector bundles

$$
\begin{aligned}
0 \rightarrow G / K \times \mathbb{R} \times V & \longrightarrow G / K \times \mathbb{R} \times V \rightarrow 0, \\
(g K, t, v) & \longmapsto \begin{cases}\left(g K, t, t \rho_{1}(g) \rho_{2}\left(g^{-1}\right) v\right) & \text { if } t \leqslant 0, \\
(g K, t, t v) & \text { if } t \geqslant 0,\end{cases}
\end{aligned}
$$

the $K$-action for the equivariant case being given by $k \cdot(g K, t, v)=\left(k g K, t, \rho_{1}(k) v\right)$.

The signficance of this definition is that elements $\delta^{G / K}(\rho) \in K^{-1}(G / K)$ admit the equivariant lifts $\delta_{K}^{G / K}(\rho) \in K^{-1}(G / K)$ by construction and pull back along $j: G \longrightarrow G / K$ to elements $\delta(\rho) \in$ $P K^{*} G$ by Theorem $7 \cdot 3$, so that $\operatorname{im}\left(j^{*} \circ \delta^{G / K}\right) \leqslant \hat{P}$ is a $K$-theoretic analogue to the space $\hat{P}_{0}$ of Definition 3.8.

Notation 7.7. In the rest of this section, we extend $i^{*}, \delta, \delta^{G / K}$, and $\delta_{K}$ to rational coefficients without further comment.

To demonstrate the utility of Theorem 1.5 we give a new proof of the (isotropy-)formality of compact, connected pairs arising from Lie group automorphisms.

Theorem 7.8. Let $(G, K)$ be a compact, connected pair. If there exists a Lie group automorphism on $G$ such that the Lie algebra of the fixed point subgroup coincides with the Lie algebra $\mathfrak{k}$ of $K$, then

1. ([Tero1, §4][Stęo2, Prop. 4.1]) G/K is formal and

2. ([GNi6]) $(G, K)$ is isotropy-formal.

Goertsches-Noshari's proof of isotropy-formality consists of a series of reductions. First, one reduces to the case where $G$ is a simple Lie group. Then it is known that all automorphisms of $G$ are conjugate through inner automorphisms to a composition $\tau \circ c_{h}$, where $\tau$ is induced by a Dynkin diagram automorphism with respect to a maximal torus $T$ of $G$ and $c_{h}$ is conjugation by some element $h \in T \cap G^{\langle\tau\rangle}$ [WG68, Lem. 5.3]. If we write $K=\left(G^{\langle\sigma\rangle}\right)_{0}$ and $H=\left(G^{\langle\tau\rangle}\right)_{0^{\prime}}$ then within $T$, the automorphisms $\sigma$ and $\tau$ both fix exactly $S=T \cap H$. It turns out that $T$ is the 
centralizer of $S$, so $S$ is a maximal torus of both $K$ and $H$ [Hel79, Lem. X.5.3, p. 492], and then by Theorem 2.2 one can replace $(G, K)$ by $(G, H)$ and $\sigma$ by $\tau$. Such $\tau$ have long been enumerated, and the remainder of the proof involves checking equality of the total Betti numbers of $G / H$ and $(G / H)^{S}$ in these cases.

As for formality, Terzić calculated $H^{*}(G / K ; \mathbb{R})$ on a case-by-case basis and picked up formality as a corollary. Stępien replaced $\sigma$ with $\tau$ as above, noted that symmetric spaces are already formal by work of Sullivan, ${ }^{9}$ and observed the remaining case, $\operatorname{Spin}(8) / G_{2} \approx S^{7} \times S^{7}$, is clearly formal.

Proposition 7.9. If $(G, K)$ is a compact, connected pair with $G$ simple and there exists a Lie group automorphism $\sigma$ on $G$ induced by a Dynkin diagram automorphism such that the Lie algebra of the fixed point subgroup coincides with the Lie algebra $\mathfrak{k}$ of $K$, then $(G, K)$ is an isotropy-formal pair.

Proof. We first consider the case where $G$ is simply-connected, so that $R(G)$ is a polynomial ring. The finite-order automorphism $\sigma$ of $G$ is induced by a graph automorphism of its Dynkin diagram and the quotient graph is the Dynkin diagram of $K$. Moreover, the rk $G$ fundamental representations of $G$ may be identified with the vertices of the Dynkin diagram of $G$ in such a way that each element of a given $\langle\sigma\rangle$-orbit restricts to the same representation of $K$. Let $\coprod_{k=1}^{\mathrm{rk} K}\left\{\rho_{k}, \sigma \rho_{k}, \cdots, \sigma^{j_{k}} \rho_{k}\right\}$ be the partition of these fundamental representations into $\langle\sigma\rangle$-orbits. Then $\operatorname{ker} i^{*}$ contains rk $G$ - rk $K$ linearly independent elements $\rho_{k}-\sigma^{j} \rho_{k}\left(1 \leqslant k \leqslant \operatorname{rk} K, 1 \leqslant j \leqslant j_{k}\right)$ The $\mathbb{Q}$-span of their images under $\delta^{G / K}$ is then a (rkG- rk K)-dimensional subspace of the Samelson space $\hat{P}$, which is itself (rk $G$ - rk $K$ )-dimensional by the dimension inequality in Theorem 2.6.(v), so the span of these elements must be all of $\hat{P}$. It follows that $(G, K)$ is formal by Theorem 2.6.(v). Note that by construction all elements in $\widehat{P}$ admit equivariant lifts. Thus $(G, K)$ is isotropy-formal by Theorem 1.5 and the discussion at the beginning of Section 7.1. In the case where $G$ is not assumed simply-connected, we may use Theorem 2.2 to reduce to the case where it is.

Note that the above proof does not use the classification of generalized symmetric spaces. Moreover it shows formality by constructing elements of the Samelson space without directly invoking Theorem A.

Remark 7.10. 1. Recall that the odd $K$-theory functor $K^{-1}$ is represented by the infinite unitary group $\mathrm{U}(\infty):=\lim _{\mathrm{U}} \mathrm{U}(n)$. Hodgkin [Hod67, pf., Cor. II.2.3] considered the map $\beta: R(G) \longrightarrow$ $K^{-1}(G)$ sending a complex $G$-representation $\rho$ to the homotopy class of $G \stackrel{\rho}{\rightarrow} \mathrm{U}(n) \hookrightarrow \mathrm{U}(\infty)$. This is in fact the same as $\delta$, but we use $\delta$ because the equivariant lift $\delta_{G}$ is slightly easier to describe than the corresponding $\beta_{G}$. The construction $\delta^{G / K}$ appears in Hodgkin's work $[\operatorname{Hod} 75, \S 10]$ on his equivariant Künneth spectral sequence.

2. The middle map of the complex of vector bundles in Definition 2 is a correction of the original definition due to Brylinski and Zhang, which Brylinski later noted was flawed and corrected. The result itself is unaffected. Discussion of the error and correction can be found in work of the second-named author [FokI4, Rmk. 2.6].

9 The proof is immediate from the result of Élie Cartan that harmonic forms on a symmetric space $M$ are closed and form a subalgebra of the de Rham algebra $\Omega^{*}(M)$ isomorphic to $H^{*}(M ; \mathbb{R})$. 


\subsection{A representation-theoretic characterization of isotropy-formality}

In this section we assume that $G$ is a compact, connected Lie group with torsion-free fundamental group unless otherwise specified.

Notation 7.11. In the rest of this section, we write $R$ for the image of the restriction map $i^{*}: R(G ; \mathbb{Q}) \longrightarrow R(K ; \mathbb{Q})$ and $I$ for its augmentation ideal $i^{*} I(G ; \mathbb{Q})$, and implicitly extend $\delta$ and $\delta_{K}$ to $\mathbb{Q}$ coefficients. We denote by $\mathcal{M}$ the $R(K ; \mathbb{Q})$-submodule $\operatorname{span}_{R(K ; \mathbb{Q})} \delta_{K}\left(\operatorname{ker} i^{*}\right)$ of $K_{K^{\text {Ad }}}^{-1}(G ; \mathbb{Q})$.

Lemma 7.12. Let $(G, K)$ be a compact, connected pair and $R=\operatorname{im} i^{*}$ as above. Then

1. both $R(G ; \mathbb{Q})$ and $R$ are integral domains finitely generated as $\mathbb{Q}$-algebras,

2. $\operatorname{Krull} \operatorname{dim} R(G)=\operatorname{rk} G$, and

3. if $\pi_{1} G$ is torsion-free, $\Omega_{R(G ; \mathbb{Q}) / \mathbb{Q}}^{1}$ is a free $R(G ; \mathbb{Q})$ module of rank equal to rk $G$.

Proof. Let $T$ be a maximal torus of $G$. Then restriction of representations induces an isomorphism $R(G) \stackrel{\sim}{\longrightarrow} R(T)^{W}[\mathrm{AH} 61, \S 4 \cdot 4]$.

1. As $R(T ; \mathbb{Q})$ is a Laurent polynomial $\operatorname{ring}$ on $\operatorname{rk} G$ generators over $\mathbb{Q}$, it follows $R(G ; \mathbb{Q}) \leqslant$ $R(T ; \mathbb{Q})$ and $R \leqslant R(K ; \mathbb{Q})$ are integral domains. As $R(T ; \mathbb{Q})$ is finitely generated over $\mathbb{Q}$, so are $R(G ; \mathbb{Q}) \cong R(T ; \mathbb{Q})^{W}[\mathrm{NSO} 2$, Thm. 2.1.4] and its quotient $R$. In fact, the connectedness hypothesis is not needed [Seg68, Cor. 3.3].

2. As $R(T ; \mathbb{Q})$ is integral over $R(G ; \mathbb{Q}) \cong R(T ; \mathbb{Q})^{W}[\mathrm{AM} 69$, Ex. 5.12], $\operatorname{Krull} \operatorname{dim} R(G ; \mathbb{Q})=$ Krull $\operatorname{dim} R(T ; \mathbb{Q})$ by going-up and lying-over. But it is clear Krull $\operatorname{dim} R(T ; \mathbb{Q})=$ rk $G .^{10}$

3. When $\pi_{1} G$ is torsion-free, $R(G ; \mathbb{Q})$ is the tensor product of a polynomial ring and a Laurent polynomial ring [Hod75, Prop. 11.1], and particularly, is a localization of a polynomial ring. But localization commutes with Kähler differentials $\left[\mathrm{ABC}^{+} 11\right.$, Cor. 12.2.16], and if $k$ is a field, then $\Omega_{k\left[x_{1}, \ldots, x_{n}\right] / k}^{1}$ is the free $k\left[x_{1}, \ldots, x_{n}\right]$-module on $d x_{1}, \ldots, d x_{n}\left[\mathrm{ABC}^{+} 11,12.2 .8\right]$.

Lemma 7.13. Let $k$ be a field of characteristic zero, $A$ an integral domain finitely generated as a $k$-algebra, and $L$ the field of fractions of $A$. Then $\operatorname{rk}_{A} \Omega_{A / k}^{1}:=\operatorname{dim}_{L} L \otimes_{A} \Omega_{A / k}^{1}=\operatorname{Krull} \operatorname{dim} A$.

Proof. By Noether normalization, $A$ is integral over some polynomial $k$-subalgebra $B$, which by going-up and lying-over has equal Krull dimension. Now Krull $\operatorname{dim} B$ is the transcendence degree of $L$ over $k$ since $B$ is a polynomial ring over $k$ and its field of fractions is $L$. But $\operatorname{tr} \operatorname{deg}_{k} L=$ $\operatorname{dim}_{L} \Omega_{L / k}^{1}\left[\mathrm{ABC}^{+}{ }_{11}\right.$, Cor. 12.3.5], and $\Omega_{L / k}^{1} \cong L \otimes_{A} \Omega_{A / k}^{1}$ since localization commutes with taking Kähler differentials $\left[\mathrm{ABC}^{+} 11\right.$, Cor. 12.2.16].

Proposition 7.14. The module $\mathcal{M}$ is of rank equal to $\mathrm{rk} G-\mathrm{rk} K$.

Proof. We have the standard exact sequence [Har13, Prop. 8.4A, Chap. II] of $R$-modules

$$
\operatorname{ker} i^{*} /\left(\operatorname{ker} i^{*}\right)^{2} \longrightarrow R \underset{R(G ; \mathbb{Q})}{\otimes} \Omega_{R(G ; \mathbb{Q}) / \mathbb{Q}}^{1} \longrightarrow \Omega_{R / \mathbb{Q}}^{1} \rightarrow 0
$$

1o Explicitly, $R(T ; \mathbb{Q})=\mathbb{Q}\left[t_{1}^{ \pm 1}, \ldots, t_{\mathrm{rk} G}^{ \pm 1}\right]$ is a localization of $\mathbb{Q}\left[t_{1}, \ldots, t_{\mathrm{rk} G}\right]$ and hence $\operatorname{Krull} \operatorname{dim} R(T ; \mathbb{Q}) \leqslant$ Krull $\operatorname{dim} \mathbb{Q}\left[t_{1}, \ldots, t_{\mathrm{rk} G}\right]=\operatorname{rk} G$ on the one hand [AM69, Ex., p. 121], and on the other $(0)<\left(t_{1}-1\right)<\cdots<$ $\left(t_{1}-1, \ldots, t_{\mathrm{rk} G}-1\right)$ is a chain of prime ideals of length $\operatorname{rk} G$ in $R(T ; \mathbb{Q})$. 
where the first map sends $\rho+\left(\operatorname{ker} i^{*}\right)^{2} \longmapsto 1 \otimes d \rho$. Extending coefficients gives an exact sequence

$$
R(K ; \mathbb{Q}) \underset{R(G ; \mathbb{Q})}{\otimes} \frac{\operatorname{ker} i^{*}}{\left(\operatorname{ker} i^{*}\right)^{2}} \longrightarrow \underbrace{R(K ; \mathbb{Q}) \underset{R(G ; \mathbb{Q})}{\otimes} \Omega_{R(G ; \mathbb{Q}) / \mathbb{Q}}^{1}}_{\mathcal{N}} \longrightarrow \underbrace{R(K ; \mathbb{Q}) \underset{R}{\otimes} \Omega_{R / \mathbb{Q}}^{1}}_{\mathcal{P}} \rightarrow 0 .
$$

Thus the kernel of $\mathcal{N} \longrightarrow \mathcal{P}$ is $\mathcal{M}$. By Lemma 7.12.3, we have $\operatorname{rk}_{R(K ; \mathbb{Q})} \mathcal{N}=$ rk $G$ and as for $\mathcal{P}$, we find

$$
\operatorname{rk}_{R(K ; \mathbb{Q})} \mathcal{P}=\operatorname{rk}_{R} \Omega_{R / \mathbb{Q}}^{1}=\operatorname{Krull} \operatorname{dim} R=\operatorname{Krull} \operatorname{dim} R(K ; \mathbb{Q})=\operatorname{rk} K,
$$

where the second equality is Lemma 7.13 , the third follows from going-up since $R(K ; \mathbb{Q})$ is integral over $K$ [Seg68, Prop. 3.2][AM69, Rmk., p. 6o], and the last is Lemma 7.12.2. By exactness, $\operatorname{rk}_{R(K ; \mathbb{Q})} \mathcal{M}=\operatorname{rk}_{R(K ; \mathbb{Q})} \mathcal{N}-\operatorname{rk}_{R(K ; \mathbb{Q})} \mathcal{P}$.

Proposition 7.15. Let $(G, K)$ be a compact, connected pair. Then $(G, K)$ is isotropy-formal if and only if $\operatorname{dim} q\left(\operatorname{ker} i^{*}\right)=\operatorname{dim} \delta\left(\operatorname{ker} i^{*}\right)=\operatorname{dim}\left(\operatorname{im} \delta^{G / K}\right)=\operatorname{rk} G-\operatorname{rk} K$.

Proof. From (6.1), the map $\operatorname{ch}_{G}$ restricts to the map $\operatorname{ker} i^{*} \longrightarrow \operatorname{ker} \rho^{*}$. Since $R(G ; \mathbb{Q})^{\wedge} \cong H_{G}^{*}$ by Theorem 5.3 , applying Lemma 5.5 in the case $A=R(G ; \mathbb{Q}), M=I(G ; \mathbb{Q})$, and $N=\mathbb{Q}$ shows that $Q R(G ; \mathbb{Q}) \longrightarrow Q H_{G}^{*}$ is also an isomorphism restricting to an injection $q \operatorname{ker} i^{*} \longrightarrow q \operatorname{ker} \rho^{*}$ of indecomposable images. The result will follow from Proposition 3.10, Theorem 1.5, and the discussion in the first paragraph of this subsection if we can show this last map is also surjective. For this, recall the $I(K)$-adic and $I(G)$-adic topologies on $R(K)$ agree [Seg68, Cor. 3.9] and complete the short exact sequence $0 \rightarrow \operatorname{ker} i^{*} \rightarrow R(G ; \mathbb{Q}) \rightarrow R(K ; \mathbb{Q}) \rightarrow 0$ at $\mathfrak{a}=I(G ; \mathbb{Q}) \triangleleft R(G ; \mathbb{Q})=A$ to conclude $\operatorname{ker} \rho^{*}=\left(\operatorname{ker} i^{*}\right)^{\widehat{l}}$. Writing $\mathfrak{b}=\operatorname{ker} i^{*} \leqslant \mathfrak{a}$, our task is to see $\left(\mathfrak{b}+\mathfrak{a}^{2}\right) / \mathfrak{a}^{2} \longrightarrow\left(\widehat{\mathfrak{b}}+\widehat{\mathfrak{a}}^{2}\right) / \hat{\mathfrak{a}}^{2}$ is surjective. It is equivalent to see $\mathfrak{b} \longrightarrow\left(\widehat{\mathfrak{b}}+\widehat{\mathfrak{a}}^{2}\right) / \widehat{\mathfrak{a}}^{2}$ is surjective, but that map factors as

$$
\mathfrak{b} \longrightarrow \mathfrak{b} / \mathfrak{a} \mathfrak{b} \stackrel{\sim}{\longrightarrow} \widehat{\mathfrak{b}} / \hat{\mathfrak{a}} \widehat{\mathfrak{b}} \longrightarrow \widehat{\mathfrak{b}} / \widehat{\mathfrak{b}} \cap \widehat{\mathfrak{a}}^{2} \stackrel{\sim}{\longrightarrow} \widehat{\mathfrak{b}}+\widehat{\mathfrak{a}}^{2} / \widehat{\mathfrak{a}}^{2}
$$

Each factor is obviously surjective except, perhaps, the second, which follows from the string of standard isomorphisms

$$
\mathfrak{b} / \mathfrak{a} \mathfrak{b} \cong \underset{A}{\mathfrak{b}} \underset{A}{\otimes} A / \mathfrak{a} \cong \mathfrak{b} \underset{A}{\otimes} \widehat{A} / \hat{\mathfrak{a}} \cong \underset{\widehat{A}}{\widehat{\mathfrak{b}}} \underset{\widehat{A}}{\widehat{\mathfrak{a}}} \cong \widehat{\mathfrak{b}} / \hat{\mathfrak{a}} \widehat{\mathfrak{b}}
$$

the penultimate isomorphism comes from Lemma $5 \cdot 5$.

Proof of Theorem B. We start by rephrasing regularity of $R$ at $I$ in numerical fashion. By Theorem 2.2 and the assumption made at the beginning of this subsection, we may assume that $G=\widetilde{G}$. By Lemma 7.13, we know $\operatorname{dim}_{R_{(0)}} R_{(0)} \otimes_{R} \Omega_{R / \mathbb{Q}}^{1}=$ Krull $\operatorname{dim} R$, and since $R$ is an integral domain finitely generated over $\mathbb{Q}$ by Lemma 7.12.1 and $I$ is a maximal ideal, this is also Krull $\operatorname{dim} R_{I}$ [AM69, Cor. 11.27]. Now, $R$ is regular at $I$ if and only if $\left[\mathrm{ABC}^{+}{ }^{11}\right.$, Cor. 3.7] these numbers equal

$$
\operatorname{dim}_{\mathbb{Q}}(R / I) \underset{R}{\otimes} \Omega_{R / \mathbb{Q}}^{1}=\operatorname{dim}_{\mathbb{Q}} \underset{R}{\mathbb{Q}} \underset{R}{\otimes} \Omega_{R / \mathbb{Q}}^{1}
$$

Tensoring the exact sequence (7.I) over $R$ with $R_{(0)}$ and $\mathbb{Q}$, we find respectively

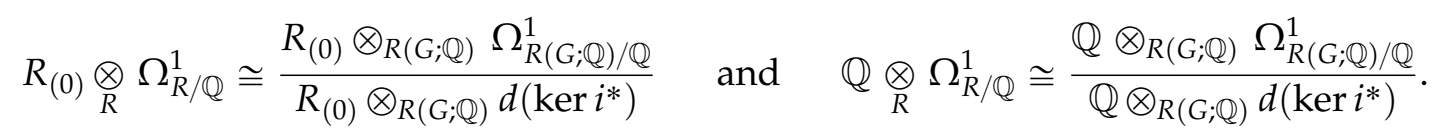


Since $\Omega_{R(G ; \mathbb{Q}) / \mathbb{Q}}^{1}$ is a free $R(G ; \mathbb{Q})$-module by Lemma 7.12.3, the numerators in $(7.2)$ are of equal dimension over their respective scalar fields, and hence we have regularity if and only if

$$
\operatorname{dim}_{R_{(0)}} R_{(0)} \underset{R(G ; \mathbb{Q})}{\otimes} d\left(\operatorname{ker} i^{*}\right)=\operatorname{dim}_{\mathbb{Q}} \underset{R(G ; \mathbb{Q})}{\mathbb{Q}} d\left(\operatorname{ker} i^{*}\right) .
$$

Now, using the isomorphisms of Theorems 7.3 and 7.4 and the observation of Remark 7.5 linking them, the left- and right-hand sides may respectively be identified with base extensions of the images of $\delta_{K}\left(\operatorname{ker} i^{*}\right)$ and $\delta\left(\operatorname{ker} i^{*}\right)$, respectively, so the equation is

$$
\operatorname{dim}_{R_{(0)}} \operatorname{span}_{R_{(0)}} \delta_{K}\left(\operatorname{ker} i^{*}\right)=\operatorname{dim}_{\mathbb{Q}} \operatorname{span}_{\mathbb{Q}} \delta\left(\operatorname{ker} i^{*}\right) .
$$

Since $R(K ; \mathbb{Q})_{(0)}$ is a field extension of $R_{(0)}$, the left-hand side is $\operatorname{rk}_{R(K ; \mathbb{Q})} \mathcal{M}$, which is equal to rk $G-$ rk $K$ by Proposition 7.14 , so finally $R$ is regular at $I$ if and only if $\operatorname{dim}_{\mathbb{Q}} \operatorname{span}_{\mathbb{Q}} \delta\left(\operatorname{ker} i^{*}\right)=$ rk $G-$ rk K. But by Proposition 7.15, this happens if and only if $(G, K)$ is isotropy-formal.

Remark 7.16. There is another way of interpreting the condition that $i^{*} R(G ; \mathbb{Q})$ be regular at $I$. As mentioned in the proof of Lemma 7.12.3, $R(G ; \mathbb{Q})$ is the ring $\mathbb{Q}\left[\rho_{1}, \cdots, \rho_{i}, t_{1}^{ \pm 1}, \cdots, t_{l-i}^{ \pm 1}\right]$. If we let $\bar{\rho}_{j}=\rho_{j}-\operatorname{dim} \rho_{j}$ and $\bar{t}_{k}^{ \pm 1}=t_{k}^{ \pm 1}-1$ be the "reduced representations" and ker $\tilde{\imath}^{*}$ is minimally generated by $\left(k_{1}, \cdots, k_{p}\right)$, then $R=\operatorname{im} i^{*}$ is regular at $I$ if and only if each of the generators $k_{1}, \cdots, k_{p}$, when written as a polynomial in the reduced representations, has nonzero linear terms, i.e., is not in $I^{2}$.

Remark 7.17. Write $\widehat{R}$ for the completion of $R$ at $I$ and $\widehat{I}$ for its augmentation ideal. Then the regularity of $R$ at $I$ is equivalent to the regularity of $\widehat{R}$ at $\widehat{I}$ since $\widehat{R}_{\widehat{I}} \cong \widehat{R}_{I}$ and [AM69, Prop. 11.24] a Noetherian local ring is regular if and only if its completion is regular. From Theorem $5 \cdot 3$, since the $I(K)$-adic and $I(G)$-adic topologies on $R(K)$ agree [Seg68, Cor. 3.9], we see $\operatorname{im}\left(H_{G}^{* *} \longrightarrow H_{K}^{* *}\right) \cong$ $\widehat{R}$, so the regularity condition in Theorem $\mathrm{B}$ can also be phrased in terms of cohomology.

Theorem B allows us to give a uniform proof of isotropy-formality (and hence formality by Theorem A) of some classes of homogeneous spaces in Examples 2.3.

Proofs for Example 2.3. (i) If $(G, K)$ is an equal-rank pair of compact, connected Lie groups, so is $(\widetilde{G}, \widetilde{K})$. The restriction map $\widetilde{\imath}: R(\widetilde{G} ; \mathbb{Q}) \rightarrow R(\widetilde{K} ; \mathbb{Q})$ is injective, so the image $R \cong R(\widetilde{G} ; \mathbb{Q})$ is a polynomial ring tensored with a Laurent polynomial ring and thus regular at $I$.

(ii) Let $(G, K)$ be a compact, connected pair such that $H^{*} G \longrightarrow H^{*} K$ is surjective. Then [Bor53, Cor., p. 179] $H_{G}^{* *} \longrightarrow H_{K}^{* *}$ is surjective as well, so $\widehat{R}=H_{K}^{* *}$, which is regular at its augmentation ideal $\hat{I}$ since it is a power series ring, so by Remark 7.17, we see $(G, K)$ is isotropyformal.

(iii) Let $(G, K)$ be a generalized symmetric pair. As in the discussion following Theorem 7.8 , we may reduce to the case $G$ is simple and simply-connected and the Lie group automorphism of $G$ is induced by a graph automorphism of its Dynkin diagram. Our proof of Proposition 7.9 shows that $\operatorname{ker}\left(i^{*}: R(G ; \mathbb{Q}) \rightarrow R(K ; \mathbb{Q})\right)$ is an ideal generated by linear combinations of reduced fundamental representations of $G$, and by Remark 7.16, im $i^{*}$ is regular at $I$. 


\subsection{Some examples}

In the following examples, $G$ is a special unitary group, and the torus subgroup $S$ we consider is one-dimensional. Thus the fundamental group of $G$ is trivial and we do not need to consider a central cover, and $R(S)$ is a principal ideal domain, so that $R(S) / / R(G)$ is a complete intersection ring and $G / S$ is formal by Theorems 6.2 and 2.6.

Example 7.18. Let $G=\mathrm{SU}(4)$ and $S=\left\{\operatorname{diag}\left(z, z^{-1}, z^{2}, z^{-2}\right): z \in S^{1}\right\}$. By Example 2.3.4, since $S$ is reflected, the pair $(G, S)$ is isotropy-formal. We can also show isotropy-formality by verifying the Shiga-Takahashi criteria of Theorem 1.4: the restriction map $\mathbb{Q}\left[c_{2}, c_{3}, c_{4}\right] \cong H_{G}^{*} \rightarrow H_{S}^{*} \cong \mathbb{Q}[s]$ assigns the universal Chern classes as elementary symmetric polynomials in $(s,-s, 2 s,-2 s)$ :

$$
c_{2} \longmapsto-5 s^{2}, \quad c_{3} \longmapsto 0, \quad c_{4} \longmapsto 4 s^{4},
$$

and so the image is $\mathbb{Q}\left[s^{2}\right] \cong H_{S}^{N}$ and the cokernel $H_{S}^{*} / / H_{G}^{*} \cong \mathbb{Q}[s] /\left(s^{2}\right)$.

We could also use Theorem B to show isotropy-formality of $(G, S)$. If $\sigma_{4}$ is the defining representation of SU(4), the reduced fundamental representations $x:=\sigma_{4}-4, y:=\wedge^{2} \sigma_{4}-6$, and $z:=\wedge^{3} \sigma_{4}-4$ generate the augmentation ideal $I(G)$ of $R(G) \cong \mathbb{Z}[x, y, z]$. If we write $R(S)=$ $\mathbb{Z}\left[t, t^{-1}\right]$ and $a=t+t^{-1}-2$, then $R(S)^{N}=\mathbb{Z}[a]$. Under the restriction $i^{*}: R(G) \longrightarrow R(S)^{N}$ of representations, the generators map as

$$
x \longmapsto a^{2}+5 a, \quad y \longmapsto a^{3}+6 a^{2}+10 a, \quad z \longmapsto a^{2}+5 a,
$$

so the image $R$ is $\mathbb{Z}\left[a^{2}+5 a, a^{3}+6 a^{2}+10 a\right]$. This is a proper subring of $\mathbb{Z}[a]=R(S)^{N}$, showing the naive modification of the Shiga-Takahashi criterion of Theorem 1.4 does not hold. However, the modification in Proposition 7.I does, for as predicted by Theorem 6.2, we have an isomorphism

$$
\begin{aligned}
R(S ; \mathbb{Q}) / / R(G ; \mathbb{Q}) & =\mathbb{Q}\left[t, t^{-1}\right] /(a) \\
& =\mathbb{Q}[t, a] /\left(a,(t-1)^{2}-a t\right) \\
& \cong \mathbb{Q}[t] /(t-1)^{2} \cong H_{S}^{*} / / H_{G}^{*}
\end{aligned}
$$

and $\left(H_{S}^{*} / / H_{G}^{*}\right)^{N} \cong\left(\mathbb{Q}[s] /\left(s^{2}\right)\right)^{N}=\mathbb{Q}$. Computing the resultant of the equations

$$
a^{2}+5 a-x=0 \quad \text { and } \quad a^{3}+6 a^{2}+10 a-y=0
$$

with respect to $a$, we find the kernel of $i^{*}$ is $\left(x-z,-x^{3}-14 x^{2}+3 x y-50 x+y^{2}+25 y\right)$, so $R \cong$ $\mathbb{Q}[x, y, z] / \operatorname{ker} i^{*}$ is regular at $I$ by Remark 7.16, and by Theorem B $(G, S)$ is isotropy-formal.

In fact, one can easily check that the $\delta^{G / S}$-images of $x-z$ and $-x^{3}-14 x^{2}+3 x y-50 x+y^{2}+25 y$ are linearly independent, so Proposition 7.15 again shows $(G, S)$ is isotropy-formal and hence formal by Theorem A. We have that

$$
K^{*}(G / S ; \mathbb{Q}) \cong \frac{\mathbb{Q}[t-1]}{(t-1)^{2}} \otimes \bigwedge_{\mathbb{Q}}\left[\delta^{G / S}(x-z), \delta^{G / S}\left(-x^{3}-14 x^{2}+3 x y-50 x+y^{2}+25 y\right)\right]
$$

The displayed exterior generators of course admit equivariant lifts.

Example 7.19. Let $G=\mathrm{SU}(3)$ and $S=\left\{\operatorname{diag}\left(z, z, z^{-2}\right): z \in S^{1}\right\}$. As $S$ is not reflected, $(G, S)$ cannot be isotropy-formal by Example 2.3.4. Alternately, we can see this using Theorem B and showing $i^{*} R(G ; \mathbb{Q})$ is not regular at $I$. Let $\sigma_{3}$ be the defining representation of $G$ so that $x=\sigma_{3}-3$ and $y=\wedge^{2} \sigma_{3}-3$ generate $I(G)$ and $R(G)=\mathbb{Z}[x, y]$. Then $i^{*}$ takes

$$
x \longmapsto 2 t+t^{-2}-3, \quad y \longmapsto 2 t^{-1}+t^{2}-3 .
$$

Computing the resultant, one finds $\operatorname{ker} i^{*}=\left(4 x^{3}+4 y^{3}-x^{2} y^{2}-6 x^{2} y-6 x y^{2}+27 x^{2}+27 y^{2}-54 x y\right)$, which nontrivially intersects $I(G)^{2}=(x, y)^{2}$, so $R$ is not regular at $I$. 


\section{References}

$\left[\mathrm{ABC}^{+}{ }_{11}\right]$ Shishir Agrawal, Eva Belmont, Zev Chonoles, Rankeya Datta, Anton Geraschenko, Sherry Gong, François Greer, Darij Grinberg, Aise Johan de Jong, Adeel Ahmad Khan, Akhil Mathew, et al. The CRing project: a collaborative, open source textbook on commutative algebra. 2011. URL: http://math.uchicago.edu/ amathew/CRing.pdf.

[Ama13] Manuel Amann. Non-formal homogeneous spaces. Math. Z., 274(3-4):1299-1325, 2013. URL: https: //link. springer.com/article/10.1007/s00209-012-1117-6, arXiv:1206.0786.

[And62] Michel André. Cohomologie des algèbres différentielles où opère une algèbre de Lie. Tôhoku Math. J. (2), 14(3):263-311, 1962. URL: https://jstage.jst.go.jp/article/tmj1949/14/3/14_3_263/_article/$\mathrm{char} / \mathrm{ja} /$.

[AB84] Michael F. Atiyah and Raoul Bott. The moment map and equivariant cohomology. Topology, 23(1):1-28, 1984. URL: https://sciencedirect.com/science/article/pii/0040938384900211.

[AH61] Michael F. Atiyah and Friedrich Hirzebruch. Vector bundles and homogeneous spaces. In Differential geometry, volume III of Proc. Symp. Pure Math., pages 7-38, Providence, RI, 1961. AMS. URL: http:// www.maths.ed.ac.uk/ aar/papers/ahvbh.pdf.

[AM69] Michael F. Atiyah and Ian G. Macdonald. Introduction to commutative algebra. Addison-Wesley, 1969.

[AS69] Michael F. Atiyah and Graeme B. Segal. Equivariant K-theory and completion. J. Diff. Geom., 3(1-18):9, 1969. URL: http://www.maths.ed.ac.uk/ aar/papers/atiyahsegal1.pdf.

[BV82] Nicole Berline and Michèle Vergne. Classes caractéristiques équivariantes. Formule de localisation en cohomologie équivariante. C. R. Acad. Sci. Paris, 295(2):539-541, Nov 1982. URL: http://gallica. bnf .fr/ ark:/12148/bpt6k62356694/f77.

$\left[\mathrm{BBF}^{+} 6 \mathrm{o}\right]$ Armand Borel, Glen Bredon, Edwin E. Floyd, Deane Montgomery, and Richard Palais. Seminar on transformation groups. Number 46 in Ann. of Math. Stud. Princeton University Press, 1960. URL: http://indiana.edu/ jfdavis/seminar/Borel, Seminar_on_Transformation_Groups.pdf.

[Bor53] Armand Borel. Sur la cohomologie des espaces fibrés principaux et des espaces homogènes de groupes de Lie compacts. Ann. of Math. (2), 57(1):115-207, 1953. URL: http://jstor.org/stable/1969728.

[BZoo] Jean-Luc Brylinski and Bin Zhang. Equivariant K-theory of compact connected Lie groups. K-Theory, 20(1):23-36, 2000. arXiv:dg-ga/9710035.

[Car14] Jeffrey D. Carlson. Equivariant formality of isotropic torus actions, I, October 2014. arXiv: 1410.5740.

[Car15] Jeffrey D. Carlson. On the equivariant cohomology of homogeneous spaces. Dissertation turned manuscript monograph, 2015. URL: http://dropbox.com/s/bczvscaxuixibac/book. pdf.

[Car16] Jeffrey D. Carlson. Macaulay2 computation demonstrating informality of a homogeneous space. 2016. URL: http://www.math.toronto.edu/jcarlson/Macaulay_CIR_test(small).txt.

[Car51] Henri Cartan. La transgression dans un groupe de Lie et dans un espace fibré principal. In Colloque de topologie (espace fibrés), Bruxelles 1950, pages 57-71, Liège/Paris, 1951. Centre belge de recherches mathématiques, Georges Thone/Masson et companie.

$\left[\mathrm{dJ}^{+}\right] \quad$ Aise Johan de Jong et al. The Stacks project. URL: http://stacks.math. columbia. edu.

[Esc92] Jost-Hinrich Eschenburg. Cohomology of biquotients. Manuscripta Math., 75(2):151-166, 1992. URL: http: //dx.doi.org/10.1007/BF02567078.

[FHTo1] Yves Félix, Steve Halperin, and Jean-Claude Thomas. Rational homotopy theory, volume 205 of Grad. Texts in Math. Springer, 2001.

[FOTo8] Yves Félix, John Oprea, and Daniel Tanré. Algebraic models in geometry, volume 17 of Oxford Graduate Texts in Mathematics. Oxford University Press, Oxford, 2008.

[Fok14] Chi-Kwong Fok. The Real K-theory of compact Lie groups. Symmetry, integrability and geometry: Methods and applications, 10:22-26, 2014. URL: http://emis.ams.org/journals/SIGMA/2014/022, arXiv:1308.3871.

[Fok17] Chi-Kwong Fok. Equivariant formality in K-theory, 2017. arXiv:1704.04796.

[Goe12] Oliver Goertsches. The equivariant cohomology of isotropy actions on symmetric spaces. Doc. Math., 17:79-94, 2012. URL: http://emis . ams .org/journals/DMJDMV/vol-17/03.pdf, arXiv: 1009.4079. 
[GN16] Oliver Goertsches and Sam Haghshenas Noshari. Equivariant formality of isotropy actions on homogeneous spaces defined by lie group automorphisms. J. Pure Appl. Algebra, 220(5):2017-2028, 2016. URL: http://sciencedirect.com/science/article/pii/S0022404915002935, arXiv:1405.2655.

[GKM98] Mark Goresky, Robert Kottwitz, and Robert MacPherson. Equivariant cohomology, Koszul duality, and the localization theorem. Invent. Math., 131(1):25-83, 1998. URL: http://math.ias.edu/ goresky/pdf/ equivariant. jour.pdf.

[GHV76] Werner H. Greub, Stephen Halperin, and Ray Vanstone. Connections, curvature, and cohomology, vol. III: Cohomology of principal bundles and homogeneous spaces. Academic Press, 1976.

[HLo7] Megumi Harada and Gregory Landweber. Surjectivity for Hamiltonian G-spaces in K-theory. Trans. Amer. Math. Soc., 359(12):6001-6025, 2007. URL: http://ams.org/journals/tran/2007-359-12/S0002-994707-04164-5.

[Har68] Bruno Harris. The K-theory of a class of homogeneous spaces. Trans. Amer. Math. Soc., 131(2):323-332, 1968. URL: http://jstor.org/stable/1994949.

[Har13] Robin Hartshorne. Algebraic geometry, volume 52 of Grad. Texts in Math. Springer, 2013.

[Hel79] Sigurdur Helgason. Differential geometry, Lie groups, and symmetric spaces, volume 8o of Pure and Applied Mathematics. Academic Press, 1979.

[Hod67] Luke Hodgkin. On the K-theory of Lie groups. Topology, 6(1):1-36, 1967. URL: https:// sciencedirect.com/science/article/pii/0040938367900109.

[Hod75] Luke Hodgkin. The equivariant Künneth theorem in K-theory. In Topics in K-theory, pages 1-101. Springer, 1975. URL: http://link. springer.com/content/pdf/10.1007/BFb0082285.pdf.

[Hopf 41 Heinz Hopf. Über eie Topologie der Gruppen-Mannigfaltigkeiten und ihre Verallgemeinerungen. Ann. of Math., 42(1):22-52, Jan 1941. URL: http://jstor.org/stable/1968985.

[JK95] Lisa C. Jeffrey and Frances C. Kirwan. Localization for nonabelian group actions. Topology, 34(2):291-327, 1995. URL: http://sciencedirect.com/science/article/pii/004093839400028J, arXiv:alg-geom/ 9307001.

[Kaneo1] Richard Kane. Reflection groups and invariant theory, volume 5 of C. M. S. Books in Mathematics. Springer, 2001.

[Kap] Vitali Kapovitch. A note on rational homotopy of biquotients. URL: http://math.toronto.edu/vtk/ biquotient.pdf.

[Kos5o] Jean-Louis Koszul. Homologie et cohomologie des algebres de Lie. Bull. Soc. Math. France, 78:65-127, 1950. URL: http://numdam.org/article/BSMF_1950_-78__65_0.pdf.

[Kos51] Jean-Louis Koszul. Sur un type d'algebres differentielles en rapport avec la transgression. In Colloque de topologie (espace fibrés), Bruxelles 1950, pages 73-81, Liège/Paris, 1951. Centre belge de recherches mathématiques, Georges Thone/Masson et companie.

[Mil62] John Milnor. On axiomatic homology theory. Pacific J. Math, 12:337-341, 1962. URL: http://msp.org/ pjm/1962/12-1/pjm-v12-n1-p31-p.pdf.

[MM65] John W. Milnor and John C. Moore. On the structure of Hopf algebras. Ann. of Math., 81(2):211-264, 1965. URL: http://jstor.org/stable/1970615?seq=1.

[Min75] Haruo Minami. K-groups of symmetric spaces I. Osaka J. Math., 12:623-634, 1975. URL: http: //ir.library.osaka-u.ac.jp/repo/ouka/all/5083/ojm12_03_06.pdf.

[NSo2] Mara D. Neusel and Larry Smith. Invariant theory of finite groups, volume 94 of Mathematical Surveys and Monographs. Amer. Math. Soc., 2002.

[Oni94] Arkadi L. Onishchik. Topology of transitive transformation groups. Johann Ambrosius Barth, 1994.

[Pit72] Harsh V. Pittie. Homogeneous vector bundles on homogeneous spaces. Topology, 11(2):199-203, 1972. URL: http://sciencedirect.com/science/article/pii/0040938372900079.

[Sal17] Bashar Saleh. Noncommutative formality implies commutative and Lie formality. Algebraic E Geometric Topology, 17(4):2523-2542, 2017. URL: http://projecteuclid.org/euclid.agt/1510841451, arXiv: 1609.02540. 
[Seg68] Graeme Segal. The representation-ring of a compact Lie group. Publ. Math. Inst. Hautes Études Sci., 34:113128, 1968. URL: http://numdam.org/item/PMIHES_1968__34__113_0.

[Shig6] Hiroo Shiga. Equivariant de Rham cohomology of homogeneous spaces. J. Pure Appl. Algebra, 106(2):173183, 1996. URL: https://sciencedirect.com/science/article/pii/0022404995000186.

[ST95] Hiroo Shiga and Hideo Takahashi. Remarks on equivariant cohomology of homogeneous spaces. Technical report 17, Technological University of Nagaoka, May 1995. URL: http://lib.nagaokaut.ac.jp/kiyou/ data/study/k17/K17_2.pdf.

[Smi67] Larry Smith. Homological algebra and the Eilenberg-Moore spectral sequence. Trans. Amer. Math. Soc., 129:58-93, 1967. URL: http://ams .org/journals/tran/1967-129-01/S0002-9947-1967-0216504-6.

[Smi95] Larry Smith. Polynomial invariants of finite groups. A. K. Peters, 1995.

[Sna71] Victor P. Snaith. On the K-theory of homogeneous spaces and conjugate bundles of Lie groups. Proc. London Math. Soc. (3), 3(3):562-584, 1971. URL: http://plms . oxfordjournals.org/content/s3-22/3/ 562.full.pdf.

[Stęo2] Zofia Stępień. On formality of a class of compact homogeneous spaces. Geom. Dedicata, 93(1):37-45, 2002. URL: http://link. springer. com/article/10.1023\%2FA\%3A1020313930539.

[Tero1] Svjetlana Terzič. Cohomology with real coefficients of generalized symmetric spaces. Fundam. Prikl. Mat, 7(1):131-157, 2001. In Russian. URL: http://mathnet.ru/php/archive.phtml?wshow=paper\&jrnid= fpm\&paperid $=556$.

[Valo8] Alessandro Valentino. K-theory, D-branes and Ramond-Ramond fields. PhD thesis, 2008. URL: https:// arxiv. org/abs/0812.0682.

[Vas69] Wolmer V. Vasconcelos. On finitely generated flat modules. Trans. Amer. Math. Soc., 138:505-512, 1969. URL: http://jstor.org/stable/1994928.

[WG68] Joseph A. Wolf and Alfred Gray. Homogeneous spaces defined by Lie group automorphisms. J. Diff. Geom, 2(1-2):77-159, 1968. URL: http://math. berkeley. edu/ jawolf/publications.pdf/paper_031.pdf.

Department of Mathematics, University of Toronto, Toronto, Ontario M5S 2E4, Canada

jcarlson@math. toronto.edu

School of Mathematical Sciences and Institute for Geometry and its Applications, University of Adelaide, Adelaide, SA 5005, Australia

chi-kwong.fok@adelaide.edu.au 